THE ASTROPHYSICAL JOURNAL, 510:726-746, 1999 January 10

(C) 1999. The American Astronomical Society. All rights reserved. Printed in U.S.A.

\title{
MAGNETOHYDRODYNAMICS OF CLOUD COLLISIONS IN A MULTIPHASE INTERSTELLAR MEDIUM
}

\author{
Francesco Miniati, ${ }^{1}$ Dongsu Ryu, ${ }^{2}$ Andrea Ferrara, ${ }^{3}$ and T. W. Jones ${ }^{1}$ \\ Received 1998 January 23 ; accepted 1998 August 18
}

\begin{abstract}
We extend previous studies of the physics of interstellar cloud collisions by beginning an investigation of the role of magnetic fields through two-dimensional magnetohydrodynamical (MHD) numerical simulations. In particular, we study head-on collisions between equal mass, mildly supersonic, diffuse clouds similar to those in our previous study. Here we include a moderate magnetic field, corresponding to $\beta=p_{g} / p_{b}=4$, and two limiting field geometries, with the field lines parallel (aligned) and perpendicular (transverse) to the colliding cloud motion. We explore both adiabatic and radiative $\left(\eta=\tau_{\text {rad }} / \tau_{\text {coll }} \simeq 0.38\right)$ cases, and we simulate collisions between clouds evolved through prior motion in the intercloud medium. In addition to the collision of evolved identical clouds (symmetric cases), we also study collisions of clouds that are initially identical but have different evolutionary ages (asymmetric cases). Depending on their geometry, magnetic fields can significantly alter the outcome of the collisions compared to the hydrodynamic (HD) case. (1) In the aligned case, adiabatic collisions, like their HD counterparts, are very disruptive independently of the symmetry. However, when radiative processes are taken into account, partial coalescence takes place even in the asymmetric case, unlike the HD calculations. (2) In the transverse case, the effects of the magnetic field are even more dramatic, with remarkable differences between unevolved and evolved clouds. Collisions between (initially adjacent) unevolved clouds are almost unaffected by magnetic fields. However, the interaction with the magnetized intercloud gas during precollision evolution produces a region of very high magnetic energy in front of the cloud. In collisions between evolved clouds with transverse field geometry, this region acts like a bumper, preventing direct contact between the clouds and eventually reversing their motion. The elasticity, defined as the ratio of the final to the initial kinetic energy of each cloud, is about $0.5-0.6$ in the cases we considered. This behavior is found in both adiabatic and radiative cases.
\end{abstract}

Subject headings: ISM: clouds - ISM: kinematics and dynamics - magnetic fields - MHD shock waves

\section{INTRODUCTION}

Our understanding of the physical processes of the interstellar medium (ISM) of the Galaxy (and of external ones) has progressed tremendously observationally and theoretically in the last decade. Part of the required effort has been stimulated by possible implications for galaxy formation in the early epochs of the universe, but it is clear that many aspects of the subject pose specific physics questions that are still unsolved, and therefore interesting to study in their own right.

That the ISM of our galaxy should present a multiphase structure has been posited for more than three decades, including in its various versions a two-, three- (and even four-) phase medium. The concept of a number of thermal phases coexisting in pressure equilibrium has now been developed further by Norman \& Ferrara (1996), who found that once turbulence is taken into account, a generalization to a continuum of phases is required.

One of the aspects that has been recognized by essentially all the authors of the above-mentioned studies as a crucial physical phenomenon in such a multiphase environment is

\footnotetext{
${ }^{1}$ School of Physics and Astronomy, 116 Church Street, SE, University of Minnesota, Minneapolis, MN 55455; min@msi.umn.edu, twj@astro.spa.umn.edu.

${ }_{2}^{2}$ Department of Astronomy and Space Science, Chungnam National University, Daejeon 305-764, Korea; ryu@canopus.chungnam.ac.kr.

${ }^{3}$ Osservatorio Astrofisico di Arcetri, Largo Enrico Fermi 5, I-50125 Firenze, Italy; ferrara@arcetri.astro.it
}

cloud collisions (CCs). Of course, the term "cloud" might literally be appropriate only for an approach that is based on a somewhat simplified thermal characterization of the ISM, whereas there is growing evidence that the dynamics of the gas, either in ordered or random/turbulent form, could govern its large-scale distribution. Nevertheless, collisions among fluid elements should in general be quite frequent and common in the ISM, and without sticking to any particular global model, the first aim of the present series of papers (Ricotti, Ferrara, \& Miniati 1997, hereafter RFM; Vietri, Ferrara, \& Miniati 1997; Miniati et al. 1997, hereafter Paper I) is to clarify the physics of such events, with special emphasis on the fate of clouds (i.e., gas clumps) and the dissipation of their kinetic energy.

The main motivation for this study (and others) is to provide a firm physical basis for global models of the ISM and galaxy evolution. Since the early paper by Oort (1954), many attempts have been made (Field \& Saslaw 1965; Habe, Ikeuchi, \& Tanaka 1981; Struck-Marcell \& Scalo 1984; Ikeuchi 1988; Vazquez \& Scalo 1989; Theis, Burkert, \& Hensler 1992; Jungwiert \& Palous 1996) to interpret the observed properties of galaxies (such as, for example, their star formation history, ISM phase evolution, and chemical evolution), as regulated by cloud self-interactions and interactions with the environment (other phases, radiation field, gravitational potential). Basically, the assumption is that the ISM exists mostly in cold gas clumps (i.e., clouds) with a spectrum of sizes, which are interacting, coalescing, forming stars (above a critical mass), fragmented again by energetic 
stellar events, and reinjected into the ISM. The model of Vazquez \& Scalo (1989), for example, includes heuristic prescriptions on the fate of collisions through a function $f_{c}$ of the relative velocity. In this way, they have been able to explain a number of interesting nonlinear behaviors as star formation bursts, whose properties are sensibly influenced by cloud interactions. Similar remarks can be made for models using cloud interaction physics to predict the phase interchange in the ISM (Habe et al. 1981; Ikeuchi 1988).

Several physical aspects of cloud collisions have been theoretically investigated in the past (Stone 1970a, 1970b; Smith 1980; Hausman 1981; Gilden 1984; Lattanzio et al. 1985; Klein, McKee, \& Woods 1995); a detailed overview of the characteristics of CCs is given in Paper I and RFM. In spite of the fact that a Galactic collision event is difficult to observe because of its infrequency, short duration, emission, and identification, a growing amount of observational evidence for the events is slowly accumulating; some examples have been found in NGC 1333 (Loren 1976), in Heiles cloud 2 (Little et al. 1978), in Draco (Rohlfs et al. 1989), and in NCP (Meyerdierks 1992). These detections often correspond to collisions involving clumps hosting a star formation region (Loren 1976). More recently, Vallée (1995) has collected convincing data for a cloud collision event toward IRAS $2306+1451$.

So far, only minor attention has been devoted to magnetized CCs. Some pioneering analytical MHD work can be found in the literature (Clifford \& Elmegreen 1983), but numerical studies have been overwhelmingly limited to hydrodynamical calculations. This is surprising, since by now magnetic fields have been detected throughout our Galaxy by a number of dedicated experiments. Observations (Spitzer 1978; Zeldovich, Ruzmaikin, \& Sokoloff 1983 and references therein) suggest that its orientation is mainly parallel to the Galactic plane, and according to some authors it becomes toroidal at high latitudes (Gomez De Castro, Pudritz, \& Bastien 1997). The magnetic field is further believed to consist of a mean systematic component and a random component. The strength of both is found to be approximately a few $\mu \mathrm{G}$. The evidence is provided by Faraday rotation, synchrotron radiation emitted by energetic electrons (cosmic rays), starlight polarization, and Zeeman effect measurements (see, e.g., Zeldovich, Ruzmaikin, \& Sokoloff 1983 for more details). Direct information about the magnetic field along the line of sight $\left(B_{\|}\right)$in Galactic diffuse clouds is obtained by observing the Zeeman splitting of the $21 \mathrm{~cm}$ radio line. With this technique, the magnetic field strength is found to range on average between 3 and $12 \mu \mathrm{G}$ in both $\mathrm{H}$ I and $\mathrm{CO}$ diffuse clouds (Myers et al. 1995). In addition, Heiles (1989) finds $B_{\|} \sim 6.4$ $\mu \mathrm{G}$ observing " morphologically distinct $\mathrm{H}$ i shells."

Recent measurements carried out by Myers \& Khersonsky (1995) have substantially improved our knowledge of the properties of magnetic fields in interstellar clouds. For the $\mathrm{H}$ I diffuse clouds in their sample, $\log x_{e}$ is found to range between -2.7 and -4.9 , where $x_{e}$ is the electron fraction. The kinetic Reynolds number, $\operatorname{Re}(=v r / v)$, and even the magnetic Reynolds number, $\operatorname{Re}_{M}\left(=v \ell / v_{M}\right)$ turn out to be large for $v \sim$ a few $\mathrm{km} \mathrm{s}^{-1}$ and $r \sim \ell \sim 1 \mathrm{pc}$. Those results validate our use of an ideal MHD code (see $\S 3.1)$ for these simulations. Large Reynolds numbers are very familiar in astrophysics, and characterize nonviscous flows. In addition, when $\operatorname{Re}_{M} \gg 1$, the field lines are well coupled with the neutrals and the magnetic flux is frozen into the fluid. Finally, the ambipolar diffusion time is given by

$$
\begin{aligned}
\tau_{\mathrm{AD}}=\left(\frac{L}{v}\right) \operatorname{Re}_{M}=2.2 & \times 10^{10} \\
& \times\left(\frac{n}{\mathrm{~cm}^{-3}}\right)^{2}\left(\frac{L}{\mathrm{pc}}\right)^{2}\left(\frac{B}{\mu \mathrm{G}}\right)^{-2} x_{e} \mathrm{yr},
\end{aligned}
$$

and therefore the field should not decay through this process in the timescales relevant for the clouds under study. In particular, this ambipolar diffusion should not affect the clouds either during their propagation through the ISM prior to, or during the collisions.

Real structures in the ISM will have complex geometries, so any attempt to model specific interactions in detail will require three-dimensional simulations. Yet ours is the first explicit MHD study of CC in which the physical effects of the presence of a magnetic field are being investigated. Because any three-dimensional studies will certainly require interpretation of very complex patterns and behaviors, we anticipate those works with an explorative two-dimensional study that should contain many of the same physical behaviors, and, being far simpler to understand, offer a practical basis for comparison. Several important and fundamentally different aspects of the physics of CCs are expected to come out of three-dimensional calculations. One important example is the appearance of Kelvin-Helmholtz (KHI) and Rayleigh-Taylor (RTI) instabilities of the cloud surface along the cylinder axial direction, which are suppressed in two-dimensional calculations. This might be particularly relevant when the magnetic field is transverse to the cloud motion, because in this case instabilities on the plane perpendicular to the cylinder axis can be suppressed by the development of an intense magnetic field at the cloud nose (Jones, Ryu, \& Tregillis 1996). This limits the time over which the two-dimensional flows are really representative. At the same time, an initial look to our preliminary result for three-dimensional single-cloud calculations (Gregori et al. 1998) reveals that part of the MHD structure relevant to CCs developed by three-dimensional clouds is qualitatively similar to that seen in two-dimensional clouds. Even though other quantitative differences must occur (see $\S 5$ ), that result certainly supports the validity of our approach, which consists of an initial explorative study of this as yet uninvestigated problem.

Since our objective is the examination of explicit MHD effects in two-dimensional cloud collisions, we try to follow as closely as possible the analogous HD simulations presented in Paper I. The plan of this paper is as follows. In $\S 2$ we give some general considerations and introduce the relevant physical quantities of the problem; in $\S 3$ we briefly describe the numerical code and the experimental setup. Section 4 is devoted to the results, which are discussed and summarized in $\S 5$.

\section{GENERAL CONSIDERATIONS AND PARAMETERS OF THE PROBLEM}

\subsection{Gas Dynamics}

In this section we review some basic aspects of purely hydrodynamical CC (HD CC), a problem already studied in great detail by previous authors (e.g., Paper I; Klein, McKee, \& Woods 1995 and references therein). The natural 
timescale for $\mathrm{CC}$ is given by

$$
\tau_{\text {coll }}=\frac{R_{c}}{v_{c}},
$$

which is approximately the time required for the shock generated by the collision to propagate across the cloud radius, $\boldsymbol{R}_{c}$. We suppose that clouds initially have a circular cross section. The main parameter determining the character of non-self-gravitating HD CCs is (Klein et al. 1995)

$$
\eta=\frac{N_{\mathrm{rad}}}{n_{c} R_{c}}
$$

where $N_{\mathrm{rad}}=n_{c} v_{c} \tau_{\mathrm{rad}}$ is the radiative cooling column density through one cloud, $n_{c}$ is the cloud number density, and $\tau_{\mathrm{rad}}$ is the cooling time (Spitzer 1978). Combining equations (2) and (3), we have $\eta=\tau_{\text {rad }} / \tau_{\text {coll }}$. Accordingly, if $\eta \leq 1$, significant radiative cooling takes place during the collision; when $\eta \gg 1$, emission processes become unimportant and the flow behaves adiabatically. In Paper I, we concluded after several low-resolution tests that the latter condition can be well represented by the weaker relation $\eta>1$. Once the two-phase model for the ISM is assumed, for a given cloud velocity these conditions imply that impacts between larger clouds are more influenced by radiative cooling (RFM; Paper I).

Since much of the basic physics of CCs has been explored using head-on events, which can provide a standard for comparison, in this paper we focus entirely on head-on CCs. In general, head-on symmetric HD CCs evolve through four main phases (Stone 1970a, 1970b); namely, compression, reexpansion, collapse, and under some circumstances, dispersal (Paper I). The occurrence of these four phases was first pointed out by Stone in his pioneering work (Stone 1970a, 1970b). It was also subsequently confirmed by high-resolution hydrodynamic calculations (Klein et al. 1995; Paper I), which allow for the highest resolution. Similar results were also found through smoothed particle hydrodynamic (SPH) calculations (e.g., Lattanzio et al. 1985; Lattanzio \& Henriksen 1988). However, the limitations of these calculations, both in resolution and in the SPH method of simulation, have led to some misinterpretations of the physics of $\mathrm{CC}$, such as the hypothesis of isothermality, as discussed in Paper I. To a certain extent, the same depiction of four phases can be drawn upon for magnetohydrodynamical CCs (MHD CCs) as well, although as we shall see there are some important differences. However, we adopt this general terminology as a useful tool to refer to the various stages of the CCs.

\subsection{Cloud Propagation through a Magnetized ISM}

The HD of a dense cloud moving into a low-density medium has been central to the work of several authors (Jones et al. 1994, 1996; Schiano, Christiansen, \& Knerr 1995; Murray et al. 1993; Vietri et al. 1997; Malagoli, Bodo, \& Rosner 1996), who generally concentrated on the growth of KHI and RTI in such conditions. We refer the interested reader to these works for an exhaustive description of this topic. When considering the motion of a cloud through a magnetized medium, new parameters in addition to those introduced so far (§ 2.1) must be considered. In particular, the initial magnetic field is completely defined by its strength and orientation. The former parameter is usually expressed in terms of

$$
\beta_{0}=\frac{p_{g}}{p_{B}}=\frac{2}{\gamma}\left(\frac{M_{\mathrm{A}}}{M}\right)^{2},
$$

where $p_{g}$ and $p_{B}=B^{2} / 8 \pi$ are the gas and magnetic pressure, and $M=v_{c} / c_{s}$ and $M_{\mathrm{A}}=v_{c} /\left[B /(4 \pi \rho)^{1 / 2}\right]$ are the sonic and Alfvénic Mach numbers, respectively. The magnetic field orientation is in general determined by two angles; in twodimensional simulations, with the field lying in the computational plane, they reduce to $\theta$, the angle between the cloud velocity and the field lines. As long as the unperturbed magnetic field is dynamically unimportant $\left(\beta_{0} \gg 1\right)$, the initial evolution of a MHD cloud is similar to a HD one. This is the case we consider below, where we adopt $\beta=4$. So, in the presence of a weak field, a stationary bow shock develops on a timescale $\tau_{\mathrm{bs}} \sim 2 \tau_{\text {coll }}$. Further, a "crushing" shock is generated and propagates through the cloud with relative speed $v_{\mathrm{cs}} \simeq v_{c} / \chi^{1 / 2}$, on a timescale (crushing time)

$$
\tau_{\mathrm{cr}}=\frac{2 R_{c}}{v_{\mathrm{cs}}}=\frac{2 R_{c} \chi^{1 / 2}}{v_{c}},
$$

where $\chi=\rho_{c} / \rho_{i}$ is the ratio of the cloud and intercloud medium densities. Finally, a low-pressure region (wake) forms at the rear of the cloud and, interacting with the converging flow reflected off the symmetry axis ( $X$-axis), generates a relatively strong tail shock. However, over time, new features develop in response to the magnetic field. Jones et al. (1996), in a two-dimensional study of individual MHD supersonic clouds, identified several of these for an adiabatic, high-Mach number cloud with modest density contrast, $\chi=10$. Mac Low et al. (1994) also studied the MHD evolution of two-dimensional individual shocked clouds, which behave in a qualitatively similar manner. Utilizing these works, in the next paragraphs we give a brief review of the main features that result from inclusion of a magnetic field during interaction with the intercloud medium.

First, the orientation of the magnetic field with respect to the direction of the cloud motion is particularly important. So far, only two extreme cases have been published; namely, cloud motion parallel (aligned) or perpendicular (transverse) to the initially uniform field. Elsewhere we present calculations with oblique magnetic fields (Miniati, Jones, \& Ryu 1998). In the aligned case, the field lines, following the flow, are swept over the cloud. As a result, those lines anchored at the cloud nose are pulled, stretched, and folded around the cloud. Eventually, these lines experience magnetic reconnection, forming new flux tubes passing around the cloud, somewhat like streamlines in a smooth flow. In this region, the magnetic field never becomes dynamically dominant, although its realignment around the cloud contributes to smoothing and therefore stabilizing the flow. On the other hand, field lines are drawn into the cloud wake. Flow in the wake stretches lines anchored in the cloud material, causing the intensity of the magnetic field to increase. This feature, referred to as the post-cloud flux rope (Mac Low et al. 1994; Jones et al. 1996), is the only one that becomes magnetically dominated $(\beta \gtrsim 0.1)$ for an aligned geometry. A similar wake region also forms in the transverse field case, where the field lines drape over the cloud, converging in its wake. In this case, however, those field lines are antiparallel across the symmetry axis. Above and 
below the symmetry axis, the field structures in the wake are initially relatively uniform, with a very sharp transition between them, corresponding to a thin current sheet. Classically, such a thin current sheet is unstable to the resistive "tearing-mode" instability (e.g., Biskamp 1993, p. 73), in which the current sheet breaks into line currents and the magnetic field reconnects across the sheet. The instability condition is that the thickness of the sheet is much smaller than its width (e.g., Biskamp 1993, p. 152 for details). Indeed, we see these sheet transitions break up into a series of closed field loops that are the signature of this instability (e.g., Melrose 1986, p. 151). This rapid modification of the magnetic field topology is often called "tearing-mode reconnection" (e.g., Melrose 1986) and typifies the reconnection that occurs in our simulations. A very clear illustration of the evolution of one such example is shown in Miniati et al. (1998, Fig. 4). Because of this behavior, the magnetic field intensities in the transverse case wakes are lower than in the aligned case. On the other hand, the field lines in front of the cloud are compressed and, more importantly, stretched around the cloud nose. In this region, unlike the previous aligned field case, reconnection does not occur for these field lines. Therefore, the magnetic field becomes very intense $\left(10^{-2} \leq \beta \leq 10^{-1}\right)$ on the cloud nose, forming a magnetic shield. As pointed out by Jones et al. (1996), the main reason for magnetic energy enhancement is the stretching of the lines as these are swept up by the cloud. The timescale for the growth of the magnetic energy is given in equation (9) of Jones et al. (1996), in the spirit of a firstorder approximation quantity, as $\tau \sim v_{c} R_{c}^{-1}=\tau_{\text {coll }}$. Soon, however, nonlinear effects become important, and a more realistic timescale, as long as two-dimensional approximation is valid, is provided by (Miniati et al. 1998)

$$
\tau \sim(\beta \chi)^{2 / 3} M^{4 / 3} \tau_{\text {coll }} .
$$

Further details on the development of the magnetic shield and its dependence on cloud characteristics can be found in Miniati et al. (1998). It is important to note that the magnetic shield acts to prevent the growth of KHIs and RTIs on the cloud surface.

When radiative losses are included, as in the HD calculations, the thermal energy of the compressed gas is lost, reducing its pressure and allowing the cloud material to be compressed to very large densities. Particularly in the transverse field case, the cloud aspect ratio (length $x$ to height $y$ ) is highly increased by this effect.

\section{NUMERICAL SETUP}

\subsection{The Code}

Our CC simulations have been performed using an ideal MHD code, based on a second-order accurate, conservative, explicit, total variation diminishing (TVD) method. Details of the MHD code are described in Ryu \& Jones (1995) and Ryu, Jones, \& Frank (1995); a brief description of some aspects concerning the inclusion of cooling and of a mass tracer is given in Appendix A. The $\boldsymbol{\nabla} \cdot \mathbf{B}=0$ condition is maintained during the simulations by a scheme similar to the constrained transport (CT) scheme (Evans \& Hawley 1988; Dai \& Woodward 1998), which is reported in Ryu et al. (1998). We have used the two-dimensional, Cartesian version of the code. The computational domain is on the $x y$ plane, and the $Z$-components of velocity and magnetic field have been set to zero.

\subsection{Grid, Boundary Conditions, and Tests}

In each CC simulation, only the plane $y \geq 0$ is included in the computational box, and reflection symmetry is assumed across the $X$-axis. The length scale is chosen for each case so that $R_{c}=1.0$, and the computational domain is adjusted to minimize boundary influences, as listed in Table 1 . Since the grid is Cartesian, our clouds are actually cylinders, with axes in the $Z$-direction. The top and right boundaries are always open. The left boundary is open in the asymmetric cases ( 3 and 4 of Table 1), and is reflective (as the bottom boundary) otherwise, when there is a mirror symmetry to the collision. With this latter choice, we are allowed to use only half of the grid, and we reduce the computational time of the calculation. Only the highest resolution calculations, characterized by 50 zones across the initial cloud radius, are presented here. Lower resolution ( 25 zones per initial cloud radius) tests were also performed in order to check for consistent behavior. It turns out that in addition to inevitable quantitative differences, for each

TABLE 1

Summary of Two-dimensional MHD Cloud Collisions Simulations

\begin{tabular}{|c|c|c|c|c|c|c|c|}
\hline \multirow[b]{2}{*}{$\operatorname{CASE}^{\mathrm{a}}$} & \multirow[b]{2}{*}{$\eta^{\mathrm{b}}$} & \multicolumn{2}{|c|}{ Clouds Ages } & \multirow[b]{2}{*}{$M_{r}^{\mathrm{c}}$} & \multirow[b]{2}{*}{$B$} & \multirow[b]{2}{*}{$\begin{array}{c}\text { GRID SIZE }^{\mathrm{d}} \\
\left(R_{c}^{2}\right)\end{array}$} & \multirow[b]{2}{*}{$\begin{array}{c}\text { END TIME }^{\mathrm{e}} \\
\left(\tau_{\text {col1 }}\right)\end{array}$} \\
\hline & & $\begin{array}{c}\mathrm{C} 1 \\
\left(\tau_{\mathrm{cr}}\right)\end{array}$ & $\begin{array}{c}\mathrm{C} 2 \\
\left(\tau_{\mathrm{cr}}\right)\end{array}$ & & & & \\
\hline $1 \ldots \ldots$ & adiabatic & 0.75 & 0.75 & 3 & $B_{x}$ & $15 \times 15$ & 75.0 \\
\hline $2 \ldots \ldots$ & 0.38 & 0.75 & 0.75 & 3 & $B_{x}^{x}$ & $15 \times 10$ & 30.0 \\
\hline $3 \ldots \ldots$ & adiabatic & 0.5 & 1.0 & 3 & $B_{x}$ & $30 \times 15$ & 22.5 \\
\hline $4 \ldots \ldots$ & 0.38 & 0.5 & 1.0 & 3 & $B_{x}$ & $30 \times 10$ & 22.5 \\
\hline $5 \ldots \ldots$ & adiabatic & 0.0 & 0.0 & 3 & $B_{y}$ & $10 \times 20$ & 30.0 \\
\hline $6 \ldots \ldots$ & adiabatic & 0.75 & 0.75 & 3 & $B_{y}$ & $20 \times 7.5$ & 30.0 \\
\hline $7 \ldots \ldots$ & 0.38 & 0.75 & 0.75 & 3 & $B_{y}$ & $20 \times 7.5$ & 30.0 \\
\hline
\end{tabular}

${ }^{a}$ All models use $\beta=4, \gamma=5 / 3, \chi=\rho_{c} / \rho_{i}=100$, and equilibrium pressure $p_{\mathrm{eq}} / k_{\mathrm{B}}=$ $1628 \mathrm{~K} \mathrm{~cm}^{-3}$. In addition, at equilibrium we have $T_{i}=7400 \mathrm{~K}$ and $n_{i}=0.22 \mathrm{~cm}^{-3}$ for the intercloud medium, and $T_{c}=74 \mathrm{~K}$ and $n_{c}=22 \mathrm{~cm}^{-3}$ inside the clouds.

${ }^{\mathrm{b}} \eta=\tau_{\mathrm{rad}} / \tau_{\mathrm{coll}}$.

c This is the relative Mach number for the cloud pair when the clouds are first set into motion. It refers to the intercloud sound speed, $c_{\mathrm{si}}$.

d The grid size is expressed in units of cloud radius. One cloud radius $R_{c}=50$ zones.

e The end time is expressed in terms of collision time $\tau_{\text {coll }}$, and represents the total time from the beginning of the collision. 
case we studied there is absolute consistency in the CC outcome. In particular, there is obvious qualitative agreement between low- and high-resolution calculations in the density distribution and magnetic field structures that form out of the CC.

\subsection{Initial Conditions}

In this section we will discuss the initial conditions for our CCs. It is worth pointing out from the very beginning that, following the results of Paper I, most of the simulated CCs involved evolved clouds, i.e., clouds that have propagated through the ISM for about $\tau_{\mathrm{cr}}$ before colliding. Initially, individual clouds have a circular cross section and uniform density, and are in pressure equilibrium with a uniform background medium; the magnetic field is also assumed to be uniform throughout the domain. The relevant parameters, whose numerical values are given below, are the density contrast $\chi$, the Mach number $M$, and the cloud radius $R_{c}$. The exact thermodynamic quantities characterizing the initial equilibrium state are not particularly important, since their memory is lost soon after the beginning of the cloud evolution; for a supersonic motion, the thermal pressure of the shocked gas and the ram pressure of the flow are dynamically far more important than the initial pressure balance. In addition, as pointed out in $\S 2.2$, the cloud motion through the intercloud medium produces a variety of features that strongly alter the initial configuration. As a result, despite the simplicity of the conditions at the onset of the cloud motion, before the collision takes place both the gas and the magnetic field are characterized by a rich structure. A comparison with the collision of two unevolved clouds is provided in the results section ( $\$ 4.2 .1$ ), to show the importance of considering prior cloud evolution in this study.

The initial values of the above parameters are the same as in Paper I and are listed below. These values are inspired by the most recent observational studies and thought to be representative of the magnetized ISM. We assume a specific heat ratio of $\gamma=5 / 3$ throughout. The intercloud medium has a density of $n_{i}=0.22 \mathrm{~cm}^{-3}$ and temperature of $T_{i}=$ $7400 \mathrm{~K}$. Clouds are characterized by a density contrast of $\chi=n_{c} / n_{i}=100$, so that the cloud density and temperature are $n_{c}=22 \mathrm{~cm}^{-3}$ and $T_{c}=74 \mathrm{~K}$, respectively, and $\tau_{\mathrm{rad}} \approx$ $3.7 \times 10^{4} \mathrm{yr}$. The sound speed in the intercloud medium turns out to be $c_{\mathrm{si}} \approx 10 \mathrm{~km} \mathrm{~s}^{-1}$, and the equilibrium thermal pressure for the ISM is $p_{\mathrm{eq}} / k_{\mathrm{B}}=1628 \mathrm{~K} \mathrm{~cm} \mathrm{~cm}^{-3}$. When initially set in motion parallel to the $X$-axis, each cloud has a Mach number $M=v_{c} / c_{\mathrm{si}}=1.5$, and therefore $v_{c} \approx 15 \mathrm{~km} \mathrm{~s}^{-1}$. Setting $R_{c}=0.4 \mathrm{pc}$ yields $\tau_{\text {coll }}=R_{c} / v_{c} \approx$ $2.6 \times 10^{4}$ yr. Since this implies $\eta=\tau_{\text {rad }} / \tau_{\text {coll }} \approx 1.4>1$, in accordance with $\S 2.1$, these collisions behave adiabatically, and therefore the cooling can be turned off. In the following discussion, collisions involving these clouds are referred to as adiabatic cases. On the other hand, setting $R_{c}=1.5 \mathrm{pc}$, we have $\tau_{\text {coll }} \approx 9.7 \times 10^{4}$ yr and therefore $\eta \approx 0.38$. These are the radiative cases. Table 2 summarizes these cloud characteristics.

With a magnetic field included, two new parameters with respect to the HD case must be determined: the field orientation and strength. The orientation is determined in two dimensions by a single parameter, namely, the angle between the initial cloud velocity and the magnetic field. We explore two cases, with the magnetic field parallel (aligned case) and perpendicular (transverse case) to the cloud velocity. For the initial strength of the magnetic field, conveniently expressed by the parameter $\beta_{0}$ (see eq. [4]), we assume $\beta_{0}=4$, corresponding to $B=1.2 \mu \mathrm{G}$. It could be argued that this value is somewhat smaller than what is usually observed. However, during the following cloud evolution, the field is stretched and amplified and in several regions becomes energetically dominant $(\beta \ll 1$ and $B>1 \mu \mathrm{G}$; see $\S 2.2$ ). In the resulting configuration, therefore, the magnetic field influence is not highly sensitive to this choice.

Initially, the Jeans length of our typical diffuse cloud is $\lambda_{\mathrm{J}} \approx 29 \mathrm{pc} \gg R_{c}$. Even though large density enhancements are produced during the compression phase in symmetric radiative collisions, $\lambda_{\mathrm{J}}$ never becomes smaller than the vertical size of the clouds. For this reason, we have neglected self-gravity throughout our calculations (see also Klein et al. 1995). Since we have concentrated on diffuse clouds, as opposed to molecular complexes, this approximation is justified.

Similarly to Paper I, we consider collisions between both radiative and adiabatic clouds. However, for brevity, we discuss here only the most significant new results for the two different cases. Table 1 summarizes the parameters of the collisions discussed below. For cases 1 and 2 in Table 1 , the cloud begins its independent evolution at $t=-\frac{3}{4} \tau_{\mathrm{cr}}$, where $t=0$ corresponds to the instant at which the bow shock of the two clouds first touch. Analogously, for cases 3 and 4 , the two clouds are placed on the grid at $t=0$ with their bow shock next to each other, after evolving for $\frac{1}{2} \tau_{\mathrm{cr}}$ and $\tau_{\mathrm{cr}}$, respectively. In these four cases, the initial magnetic field was aligned with the cloud motion, whereas for cases 5-7 it was transverse. Case 5 is the only nonevolved calculation we present-by this we mean a uniform cloud of

TABLE 2

Clouds CharaCteristics ${ }^{\mathrm{a}}$

\begin{tabular}{ccccc}
\hline \hline$\eta^{\mathrm{b}}$ & $\begin{array}{c}R_{c} \\
(\mathrm{pc})\end{array}$ & $\begin{array}{c}\tau_{\text {coll }}=R_{c} / v_{c} \\
(\mathrm{yr})\end{array}$ & $\begin{array}{c}\tau_{\mathrm{cr}}=2 R_{c} \sqrt{\chi} / v_{c} \\
(\mathrm{yr})\end{array}$ & $\begin{array}{c}\tau_{\text {rad }}=(3 / 2) k T / n \Lambda \\
(\mathrm{yr})\end{array}$ \\
\hline Adiabatic $\ldots \ldots . .$. & 0.4 & $2.6 \times 10^{4}$ & $5.3 \times 10^{5}$ & $\infty^{\mathrm{c}}$ \\
$0.38 \ldots \ldots \ldots \ldots$ & 1.5 & $9.7 \times 10^{4}$ & $2.0 \times 10^{6}$ & $3.7 \times 10^{4}$ \\
\hline
\end{tabular}

${ }^{a}$ All models use $\beta=4, \gamma=5 / 3, \chi=\rho_{c} / \rho_{i}=100$, and equilibrium pressure $p_{\mathrm{eq}} / k_{\mathrm{B}}=1628 \mathrm{~K} \mathrm{~cm}^{-3}$. In addition, at equilibrium we have $T_{i}=7400 \mathrm{~K}$ and $n_{i}=$ $0.22 \mathrm{~cm}^{-3}$ for the intercloud medium, and $T_{c}=74 \mathrm{~K}$ and $n_{c}=22 \mathrm{~cm}^{-3}$ inside the clouds.

${ }^{\mathrm{b}} \eta=\tau_{\mathrm{rad}} / \tau_{\mathrm{coll}}$.

c The actual value of the cooling time for the smaller cloud is also $\tau_{\mathrm{rad}}=3.7 \times 10^{4}$ yr. However, as explained in the text, since $\eta>1$, the cooling does not affect the collision of these clouds, and therefore it has been turned off during the simulations. For this reason, we have set $\tau_{\text {rad }}=\infty$ in the table. 
circular cross section placed on the grid in such a way that its boundary is only 2 zones from the (reflecting) $Y$-axis at $t=0.0$. Finally, in cases 6 and 7, the cloud starts its evolution at $t=-\frac{3}{4} \tau_{\mathrm{cr}}$ and is then treated as in cases 1 and 2 , respectively. Animations of each simulation have been posted on our web site at the University of Minnesota. ${ }^{4}$

\section{RESULTS}

\subsection{Aligned Field (BX, Cases 1-4)}

In the aligned field case, MHD CCs show many similarities with HD CCs. These are illustrated in Figure 1, where the evolution of various MHD cloud integral properties are plotted as a function of time. The curves of kinetic and thermal energy, as well as $Y_{\mathrm{cm}}$ (the $Y$-coordinate of the center of mass), closely resemble those for HD clouds in Figures 8 and 9 of Paper I, and all the phases characteristic of a HD CC (see $\S 2.1$ and Paper I) are clearly seen. In general, a stronger compression is generated when the clouds have mirror symmetry across the impact plane, and only a weak reexpansion takes place in radiative cases, because most of the thermal energy is radiated away. The top right panel of Figure 1 displays the total magnetic energy. Since its variations are related to compression and/or stretching of the field lines, this quantity gives an approximate measure of the overall interaction between the magnetic field and the gas. In the adiabatic cases (Fig. 1, solid and dotted lines, respectively) the large expansion undergone by the cloud gas produces both significant stretching and compression of the field lines. As a result, during the reexpansion phase, the total magnetic energy increases by about $30 \%$. On the other hand, in all radiative cases the total magnetic energy suffers only slight variations.

We now begin specific comparison, considering the adiabatic collision of two evolved identical clouds with aligned fields.

\subsubsection{Symmetric Cases}

The evolution of case 1 is reported in Figures $2 a$ and $2 b$, which show the field line geometry (left panels) and density distribution (right panels) at four different times $\left(t / \tau_{\text {coll }}=\right.$ $4.5,24,48.75$, and 75 ). As complementary quantitative information, Figures 3, 4, and 5 provide, for the same times, cuts along the primary axes of density, thermal and magnetic pressure, respectively. Lines in these figures correspond to $t=4.5 \tau_{\text {coll }}$ (solid lines), $t=24 \tau_{\text {coll }}$ (dotted lines), $t=48.75 \tau_{\text {coll }}$ (dashed lines), and $t=75 \tau_{\text {coll }}$ (dot-dashed lines). Cuts in the top panels are along the $X$-axis, while those in the bottom panels are along the $Y$-axis.

As suggested above, the evolution of the CC is substantially unaffected by the magnetic field. Indeed, the CC goes through the four phases mentioned in $\S 2.1$, as in the HD case. For the compression and reexpansion phase, there is a close quantitative correspondence, as can be inferred from a comparison of Figure 3 (solid and dotted lines) and Figure 2 of Paper I (although lines in the two figures do not correspond exactly to the same time). This is not unexpected, however, since the compression and reexpansion phases are dominated by the high pressure of the shocked gas.

During the reexpansion phase, a thin shell of dense $\left(4 \rho_{i} \leq \rho \leq 10 \rho_{i}\right)$ cloud gas forms behind the reverse shock

\footnotetext{
${ }^{4}$ Animations available on our web site: http://www.msi.umn.edu/ Projects/twj/mhd-cc.
}

of the expanding material. A long finger appears on the $X$-axis as the reexpansion finds an easy way through the flux rope, where the density and the pressure are quite low (Mac Low et al. 1994; Jones et al. 1996). In addition, in contrast to the HD case, the shell boundaries in Figure $2 a$ (bottom left) are quite sharp. This difference has an important physical basis. In fact, although the initial magnetic field does not have sufficient strength to inhibit the onset of KHI (Chandrasekhar 1961), nevertheless it is able to reduce and eventually stop its growth. In fact, as the magnetic field lines, frozen in the gas, get stretched in the turbulent flow, their strength is increased until during eventual reconnection they redesign the flow pattern to a more stable configuration. The criterion for this field dominance is that the local Alfvénic Mach number falls to order unity or less (Chandrasekhar 1961; Frank et al. 1996; Jones et al. 1997). This is evinced by the presence of several field-line loops on the external side of the gas shell (Fig. 2a, lower left panel). Inside the shell, the magnetic field intensity has severely dropped. According to Figure 5, the magnetic energy density (dotted line) has been reduced with respect to its initial value (solid line) by a factor ranging from $10^{2}$ to $10^{4}$. This cannot be accounted for by expansion alone; complex reconnection processes at the beginning of the reexpansion phase are responsible as well.

The collapse phase (at $t \sim 18.75 \tau_{\text {coll }}$ ) is chaotic and turbulent; in and around the cloud gas the density distribution is rather clumpy, and the magnetic field has a tangled structure (Fig. 2b, top panels, and Figs. 3 and 5, dashed lines). This situation will persist until the end of the simulation (Figs. 3 and 5, dot-dashed lines). It is during this phase that the magnetic field produces a qualitative change in the evolution of the CC. In Paper I, we showed that during the HD collapse phase the reverse shock propagates toward the inner region of the expanded cloud gas, whereas the external layer is shredded by KHIs and RTIs. At the end, numerous filaments fill a region of about the same size and shape as the shell at its maximum extent. In the present case, on the other hand, the magnetic field lines that have been stretched by the vertical expansion of the gas shell begin to relax, accelerating the gas at the top of the shell (near the $Y$-axis) toward the $X$-axis (Fig. $2 b$, top panels). At the end of the collision $\left(t=75 \tau_{\text {coll }}\right)$, the initial cloud material is confined in a layer beneath the relaxed magnetic field lines, with a mean density of $\rho \sim 4 \rho_{i}$ (Fig. $2 b$, bottom panels), and is still laterally expanding. Within this new structure, with a thickness of several initial cloud radii, we find a clumpy density distribution (Fig. 3, dot-dashed lines) and a weak, irregular magnetic field (Fig. 5, dot-dashed lines). Outside it, on the other hand, the magnetic field has the same initial configuration but greater strength, often twice as much as its initial value (Fig. 5, bottom panel, dot-dashed line).

The analogous radiative case (case 2) is illustrated in Figure 6, and its quantitative properties are plotted in Figure 7. Again, an aligned magnetic field does not seem to influence the collision. As in the HD case, efficient radiative cooling allows a much higher gas compression (Fig. 7, top panel, solid line) and inhibits the strong reexpansion that would be driven by the high pressure of the shocked gas (Fig. 7, bottom panel, solid line). During these phases the clouds coalesce, generating a well-defined high-density round structure about twice as large as the initial single cloud (this can be inferred from the position of the sudden drop in the dotted line in top panel of Fig. 7). No collapse 

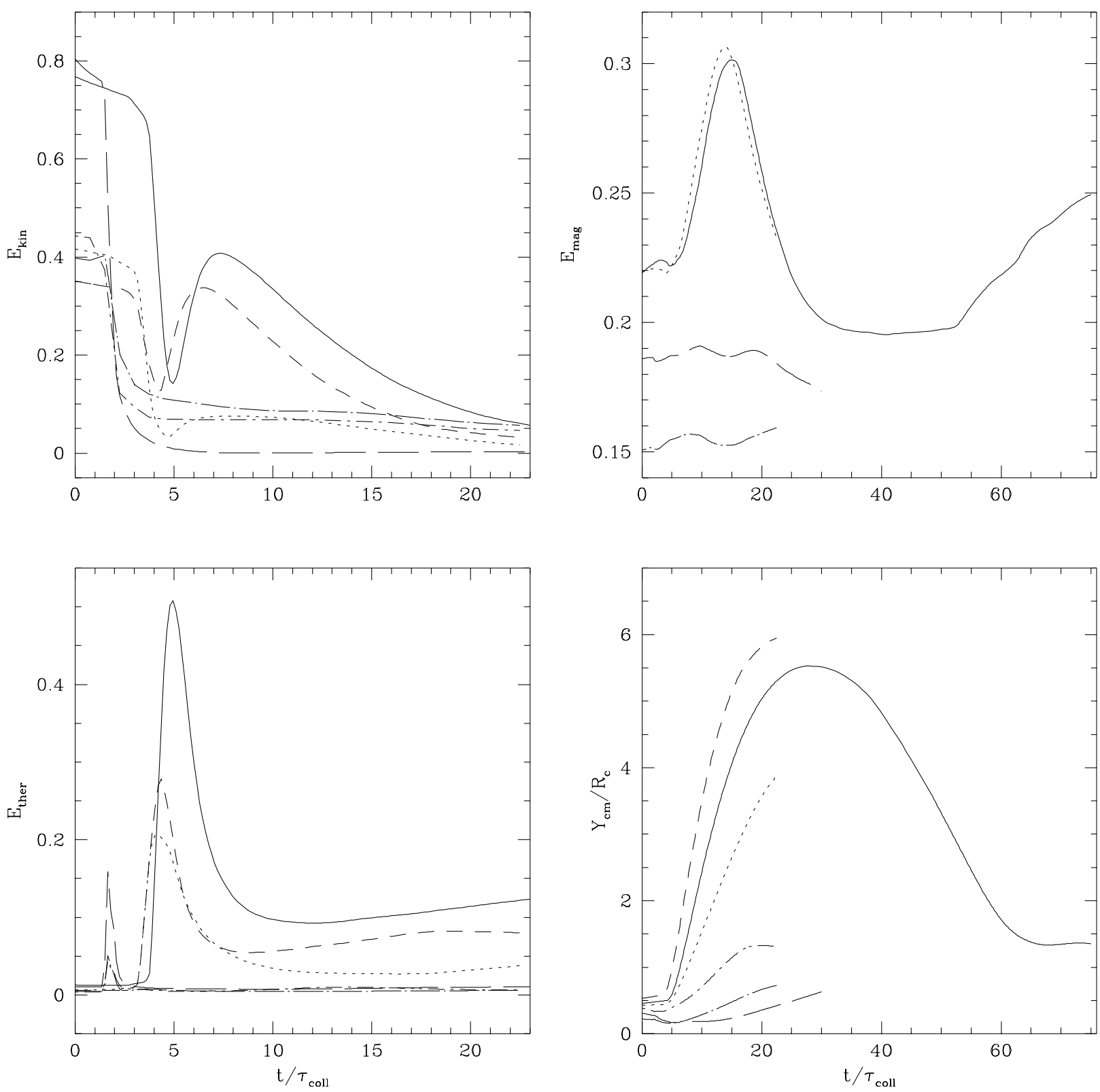

FIG. 1. $-B X$ plots. Panels show the evolution of kinetic energy (top left), thermal energy (bottom left), and $Y_{\mathrm{cm}}$ coordinates (bottom right) of the colliding clouds and of the total magnetic energy within the grid (top right) as a function of time, for the aligned field cases. For cases 1 and 2, the two colliding clouds are identical to each other, so only one is displayed. In the first three panels, solid line shows case 1, long-dashed lines show case 2 . Dotted and short-dashed lines show, respectively, clouds C1 (left) and C2 (right) in case 3. Dot-short-dashed and dot-long-dashed lines show, respectively, clouds C1 (left) and C2 (right) in case 4. In the last panel (top right), the solid lines show case 1, dashed line case 2, dotted line case 3, and dot-long-dashed line case 4. The kinetic and thermal total magnetic energy in these plots has been normalized to the sum of the cloud energy (kinetic + thermal + magnetic) and the background magnetic energy at the beginning of the simulation. Therefore, with respect to Paper I, the vertical scale of the kinetic and thermal energy of the cloud is reduced by a factor of 1.18 .

phase ever happens. In addition, a narrow jet of gas, also characteristic of radiative HD CC, is formed and extends along the $Y$-axis. As it propagates transverse to the magnetic field lines, a high-density spot is created at the leading edge. However, those features are usually unimportant, because they only involve a negligible fraction of the total mass. Of more interest is rather the final fate of the mentioned structure. At the end of our simulation $\left(t=30 \tau_{\text {coll }}\right)$, its edge near the $X$-axis is still expanding along the $X$-axis with a speed of $v \sim 0.1 c_{\mathrm{si}}$. According to the three density cuts in the top panel of Figure 7, the cloud edge (located at the sharp drop in each line) has been expanding at roughly the same speed $\left(\sim 0.1 c_{\mathrm{si}}\right)$ throughout the evolution. Based on this velocity, we can, therefore, estimate the time $\tau_{\alpha}$ for the density of the new structure to drop by a factor $\alpha$. Assuming that only one dimension of the cloud volume increases (at the speed of $0.1 c_{\mathrm{si}}$ ) as a result of its expansion, we have $\tau_{\alpha} \simeq \alpha R_{c} /\left(0.1 c_{\mathrm{si}}\right)=\alpha 10 M \tau_{\text {coll }}=\alpha 1.5 \times 10^{6} \mathrm{yr}$ (we used $\tau_{\text {coll }} \sim 10^{5} \mathrm{yr}$ from $\S 3.3$ ). Since inside the new structure $\rho \sim 40-80, \alpha$ can be as large as 5-10, and $\tau_{\alpha}$ is comparable to the time between two cloud collisions.

\subsubsection{Asymmetric Cases}

As in Paper I, we produce a simple asymmetric CC by colliding clouds with the same initial mass, velocity, and radius, but evolved individually for a different time interval 

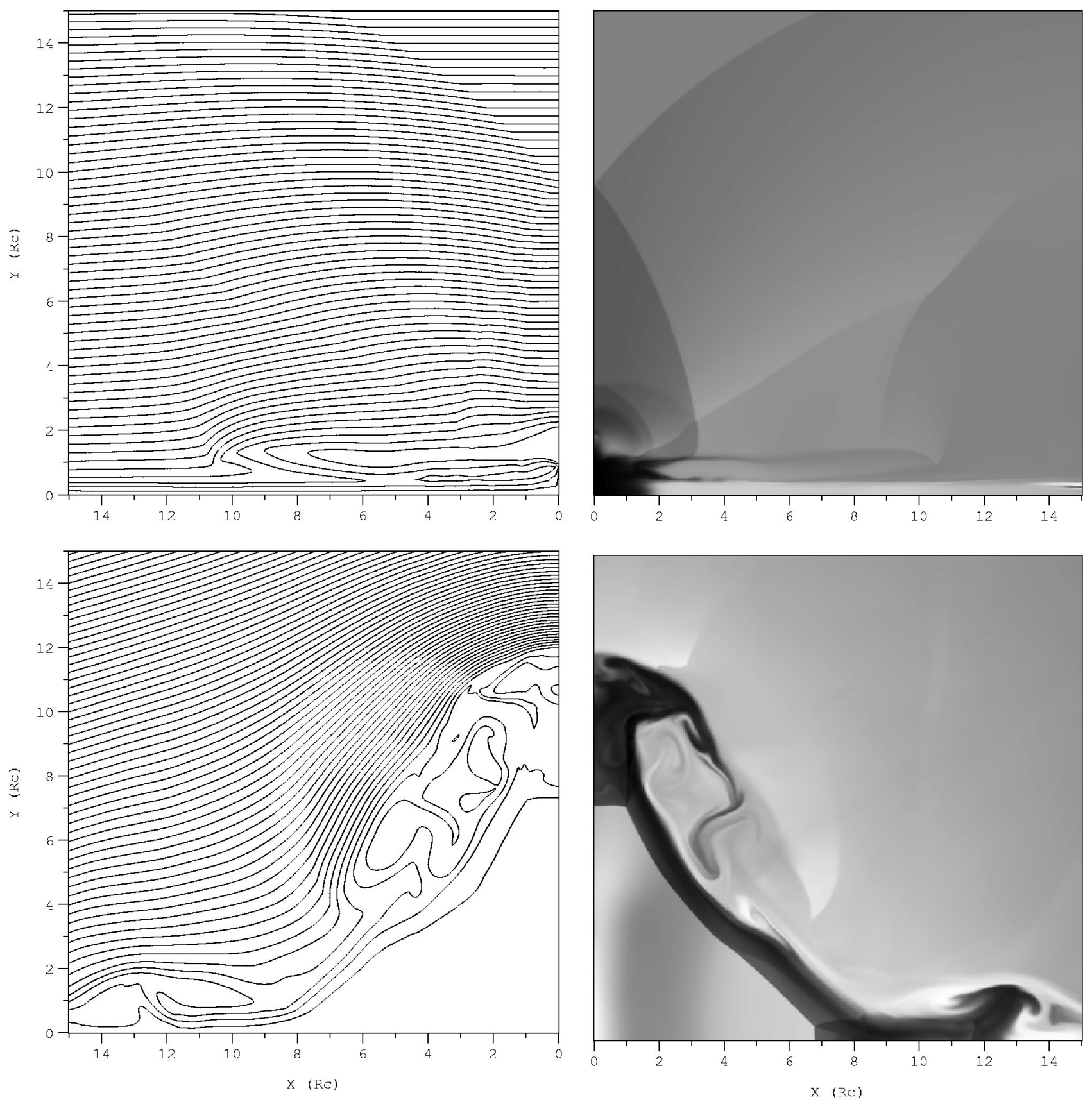

Fig. 2.-From top to bottom, the four pairs of panels show field-line geometry $(l e f t)$ and density distribution at $t / \tau_{\text {coll }}=4.5,24,48.75$, and 75 . Coordinates are expressed in units of cloud radii, $R_{c}$. Field lines are contours of the magnetic flux, and correspond to a change in the latter by a factor of 7 . Density figures are inverted gray-scale images of $f(\rho)=\rho /(1+\rho)$. The top two panels show the compression phase $\left(t=4.5 \tau_{\text {coll }}\right)$, with field lines swept up by the vertical outflow at the symmetry plane of the collision. The second two panels shows the reexpansion phase $\left(t=24 \tau_{\text {coll }}\right)$, with the formation of a long finger at the back of the cloud, a large spot near the symmetry axis, and a thin shell-like structure. Numerous closed field loops witness the occurrence of reconnection processes. The next two panels capture the collapse $\left(t=48.75 \tau_{\text {coll }}\right)$ of the large spot pushed by relaxing field lines. The bottom two panels show the end of the collision $\left(t=75 \tau_{\text {coll }}\right)$, with the formation of a thick layer of cloud gas and low magnetic energy.

before they collide. By contrasting these with mirror symmetric cases, we can begin to see properties that are symmetry dependent. In the following cases (case 3 and 4), the two clouds have been evolved for about $\frac{1}{2} \tau_{\mathrm{cr}}$ and $\tau_{\mathrm{cr}}$, respectively (Table 1).

The evolution of the adiabatic asymmetric $\mathrm{CC}$ resembles the analogous symmetric case 1 . Figure 8 shows the field lines (top panel) and the density distribution (bottom panel) at the end of the reexpansion phase $\left(t=22.5 \tau_{\text {coll }}\right)$. We can identify a relatively dense shell $\left(\rho \sim 5 \rho_{i}\right)$ with a large clump of gas on top of it, as well as loops of magnetic field lines generated by reconnection events. Some new features appear, however, as a result of the broken symmetry. One example is the long tail of cloud gas on the right-hand side of Figure 8, produced by the unbalanced momentum distributions in the two clouds along the $X$-axis. Nevertheless, they are not so significant as to alter the general character of this CC with respect to case 1. Therefore, we expect that the collapse phase and the remaining following evolution of this case will not differ qualitatively from case 1 . We also 

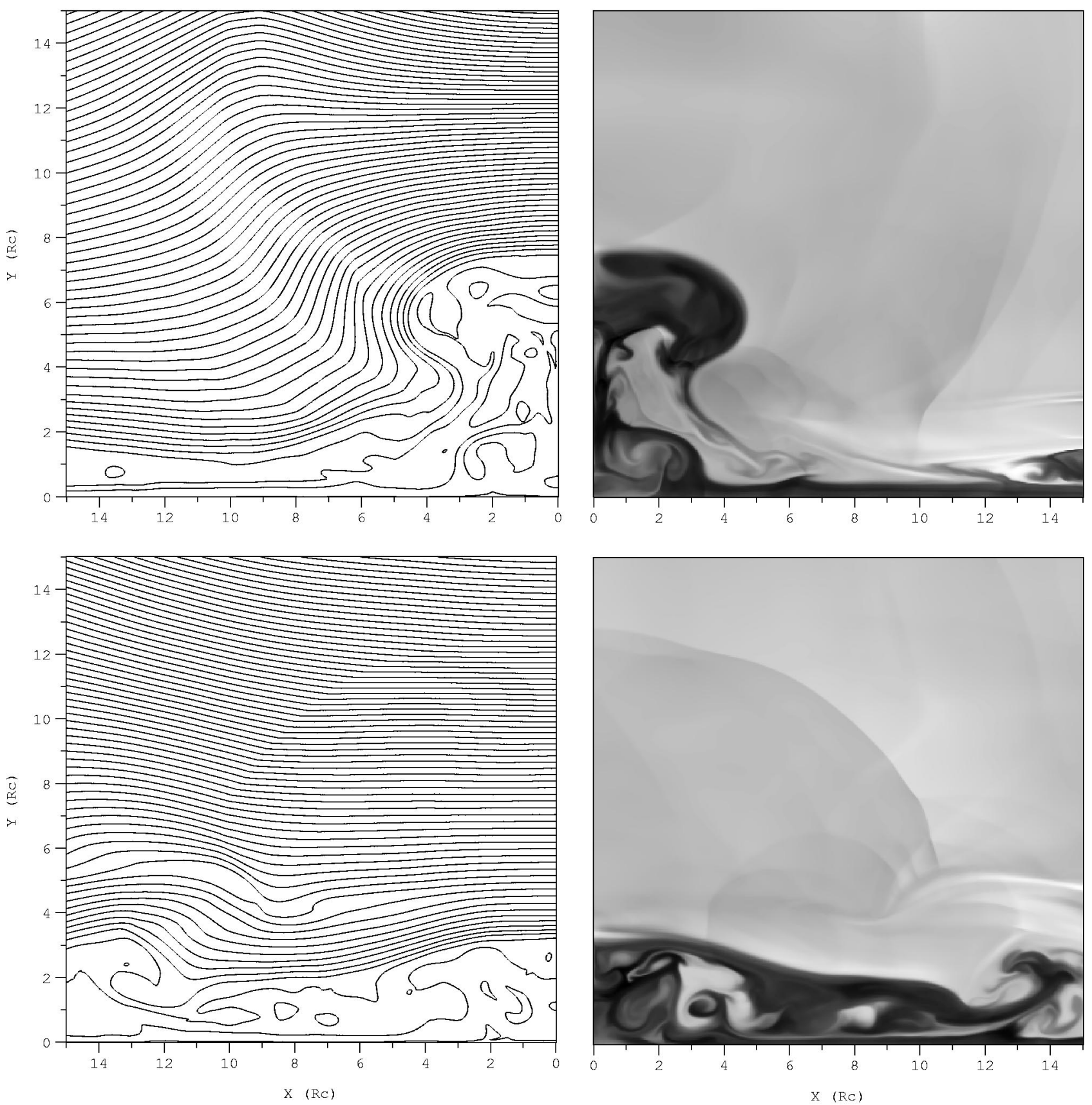

FIG. 2.-Continued

point out that as long as the asymmetry does not prevent the development of a reexpansion phase, case 1 can be considered as qualitatively well representative of adiabatic MHD CC with a magnetic field aligned to the initial cloud motion.

The radiative case (case 4), shown in Figures $9 a$ and $9 b$, presents new and interesting insights. The most crucial part of the evolution is represented by the cloud interaction during the compression phase. During that phase, the older and more compact cloud $(\mathrm{C} 2)$, moving from the right, attempts to plow through the other cloud (C1) (Fig. 9a; $\left.t=6.75 \tau_{\text {coll }}\right)$. Some of the features that are visible in case 2 , such as the vertical jet of gas, can also be recognized here if one carefully accounts for the distortions arising from the asymmetry.
However, we give particular attention to the new feature on the $X$-axis toward the left of Figure $9 a\left(X \simeq-5 R_{c}\right)$. This is a compact clump, with a density of about $5 \times 10^{2} \rho_{i}$ and velocity along the $X$-axis $v_{x} \sim-\frac{1}{2} c_{\mathrm{si}}$. At the end of the simulation $\left(t=22.5 \tau_{\text {col1 }}\right)$, it has expanded and is still moving to the left along the $X$-axis (Fig. $9 b$ ). At this time, the "mass tracer" variables allow us to conclude that despite the large prevalence of gas from the more compact cloud (C2), the new clump is the result of a partial coalescence of the two initial clouds. Its mass is about $10 \%$ larger than the initial mass of either cloud. Its density varies between $15 \rho_{i}$ and $100 \rho_{i}$, and the velocity pattern suggests that it is expanding along the $X$-axis with $v \sim c_{\mathrm{si}} / 6$. By the same argument, at the end of $\S 4.1 .1$ we can therefore conclude that for this case, $\tau_{\alpha} \simeq \alpha 9 \times 10^{5} \mathrm{yr}$. Again, before the cloud disperses in 

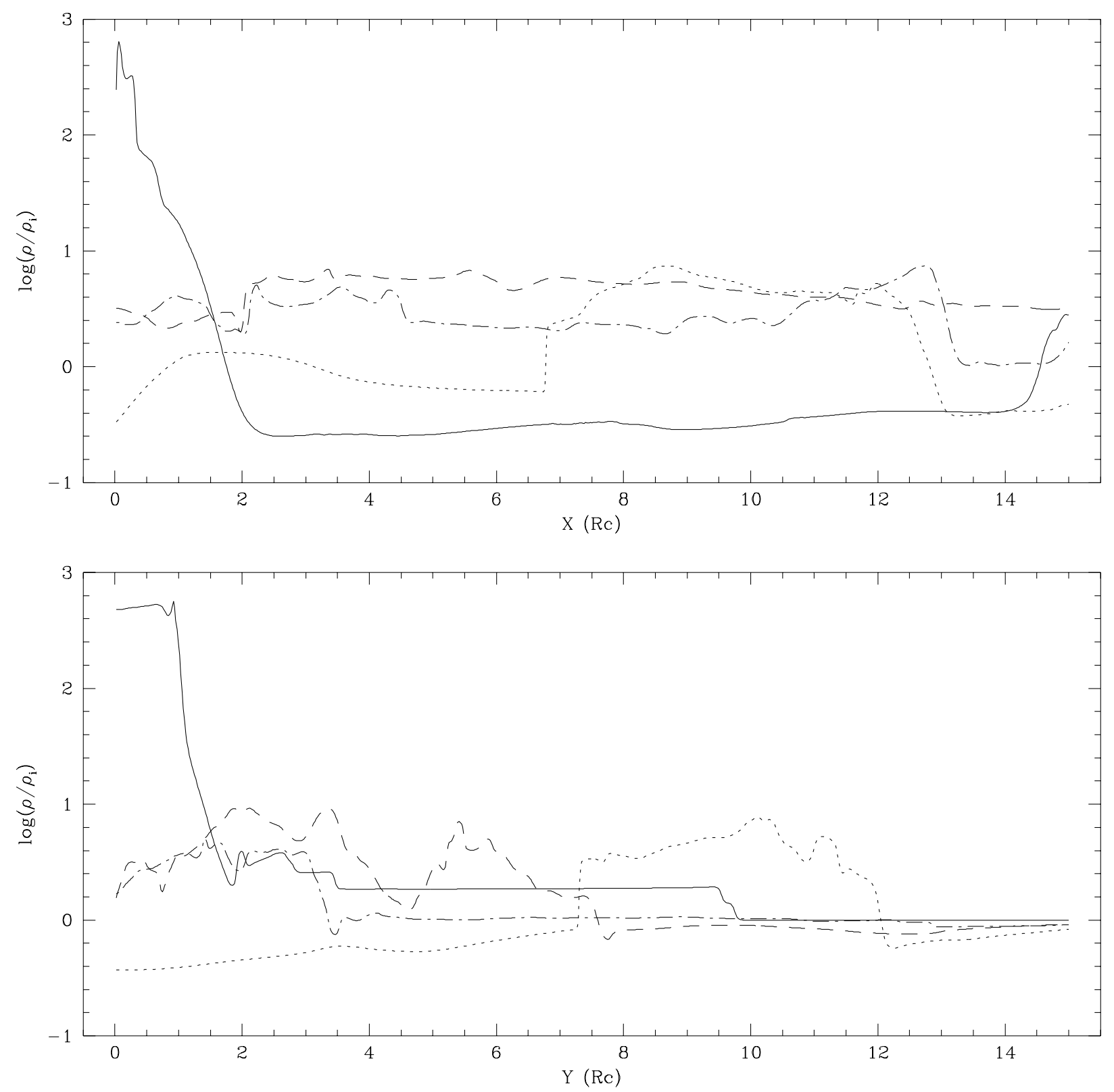

FIG. 3. - Log density cuts through the grid along the $X$-axis $\left(y=0.3 R_{c}\right.$, top panel $)$ and along the $Y$-axis $\left(x=0.1 R_{c}\right.$, bottom panel) for case 1 . Solid lines show $t=4.5 \tau_{\text {coll }}$, dotted lines show $t=24 \tau_{\text {coll }}$, dashed lines show $t=48.75 \tau_{\text {coll }}$, and dot-dashed lines show $t=75 \tau_{\text {coll }}$.

the background medium, the newly formed clump is likely to undergo another CC. The remainder of C1's gas (Fig. $9 b$, feature to the right), moving transversely to the magnetic field, has formed a long filamentary structure and has created a sharp cusp in the field lines.

This result strongly differs from the analogous purely HD calculation, where we found that the collision produced a large, low density contrast filamentary structure, eventually fading into the background gas.

\subsection{Transverse Field (BY, Cases 5-8)}

We start this section by presenting the case of a $\mathrm{CC}$ between two unevolved clouds (case 5). This calculation is mostly intended to provide a reference case when studying evolved CCs, and thus to emphasize the importance of initial conditions in calculations of this type.

\subsubsection{Unevolved Adiabatic Collision}

The results of this calculation (case 5) are shown in Figure 10 , where two density images are superposed on field lines. The left panel captures the reexpansion phase at $t=7.5 \tau_{\text {coll }}$; it closely resembles the analogous HD case 1 of Paper I, and no significant difference from case 1 (of this paper), where the magnetic field was aligned to the motion, can be pointed out. This means that no major role is played by the magnetic field, as has been the case so far for all adiabatic CCs. The shell density has typical values $\left(\sim 10 \rho_{i}\right)$, and the thermal pressure drives the reexpansion. RTIs develop on the shell surface near the $Y$-axis. However, as we show below, at the end of the collision, a thick layer, qualitatively similar to that formed in cases 1 and 3, forms along the $Y$-axis. We also point out that the evolution of the total magnetic energy (Fig. 11, bottom left panel, dashed line) is 

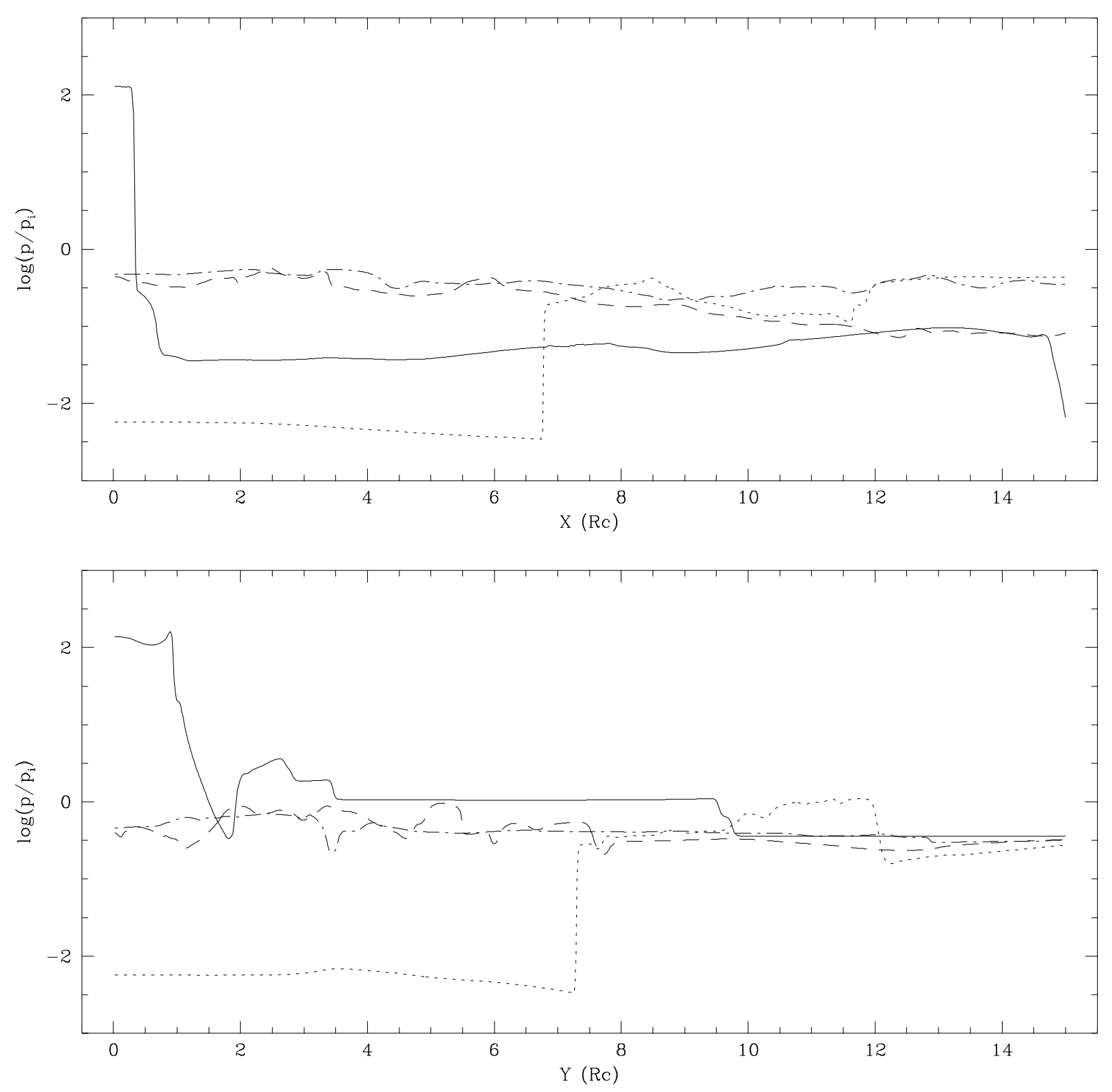

FIG. 4.- Same as Fig. 3, but for the logarithm of thermal pressure

reliable only up to $t \sim 9 \tau_{\text {coll }}$, which marks the exit of the forward blast shock from the right boundary of the grid, along with the consequent outflow of magnetic energy. The right panel image in Figure 10 is from $t=30 \tau_{\text {coll }}$. RTIs, aided by the formation of a slow, switch-off shock, which aligns the field with the expansion of the shell, have formed a large finger expanding almost parallel to the $Y$-axis.

\subsubsection{Evolved Symmetric Cases}

As mentioned in $\S 2.2$ and discussed by Jones et al. (1996), an individual cloud moving transverse to a magnetized intercloud medium develops a region of strong magnetic field known as the magnetic shield. That feature dominates the collisions of such evolved clouds from the start of their encounter. Consequently, magnetic field effects dominate the evolution in cases 6 and 7, in striking contrast to case 5 . It follows that meaningful simulations of CCs may depend on understanding the field geometry in their surroundings and allowing self-consistent magnetic structures to evolve before collisions take place.

Case 6 is shown in Figures $12 a$ and 12b, which include both field line geometry and density images superposed on the velocity field. In addition, the solid lines in Figure 11 show the usual time evolution of integral quantities. Peaks in the thermal energy and corresponding valleys in the $Y_{\mathrm{cm}}$ curve are signatures of the bow shock precompression and the "collision" undergone by the cloud. Note the simultaneous kinetic energy decrease and the magnetic energy increase, primarily resulting from cloud interaction with the magnetic field (Miniati et al. 1998) at the beginning of the simulation and from the collision event later on.

As the cloud approaches the collision plane ( $Y$-axis) in Figure $12 a$ (bottom panel), the field lines are highly compressed (top panel), generating a strong repulsive force opposite to the cloud motion. Eventually, all the cloud kinetic energy is converted into magnetic form and stored 

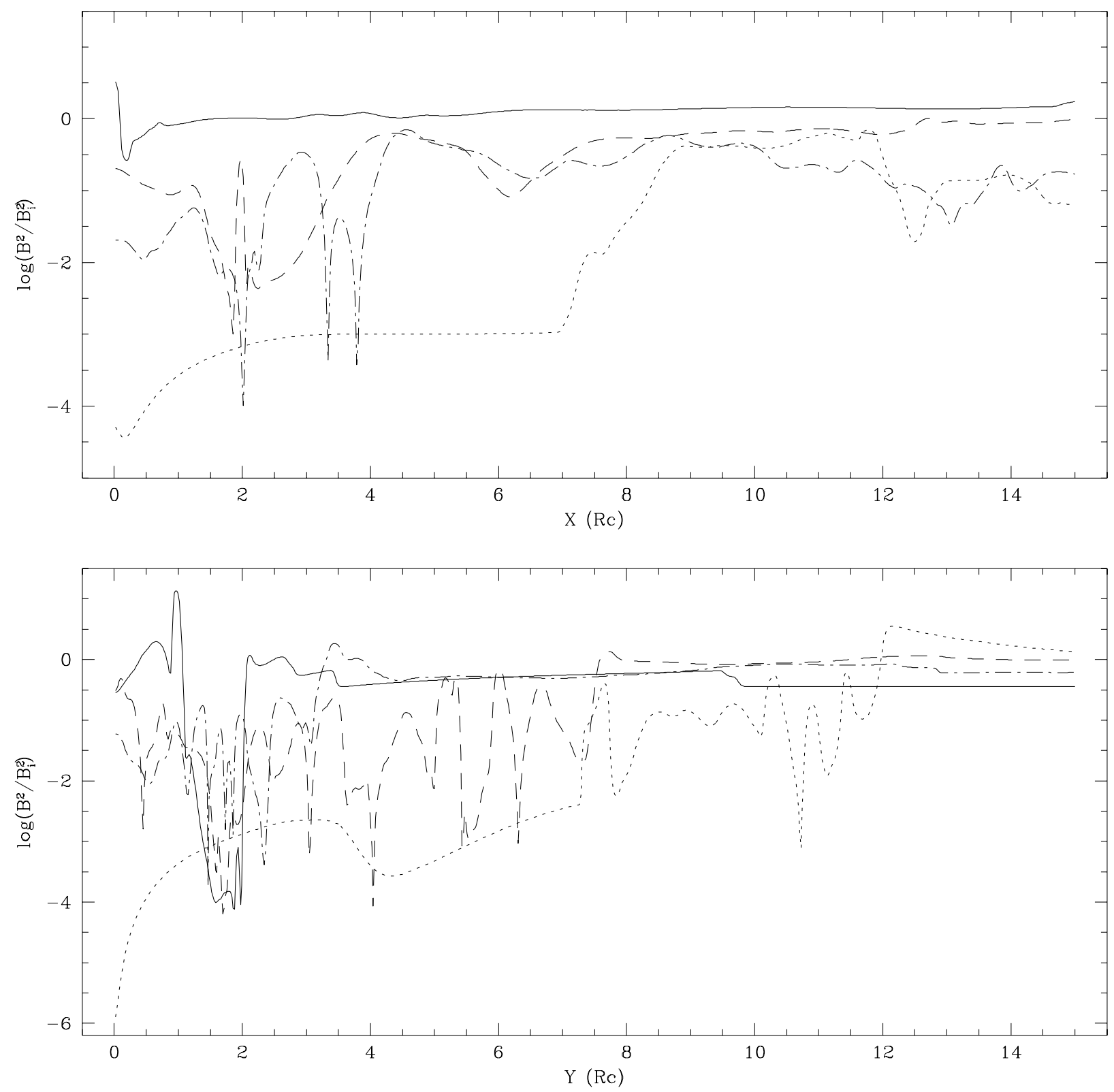

FIG. 5.-Same as Fig. 3, but for the logarithm of magnetic energy
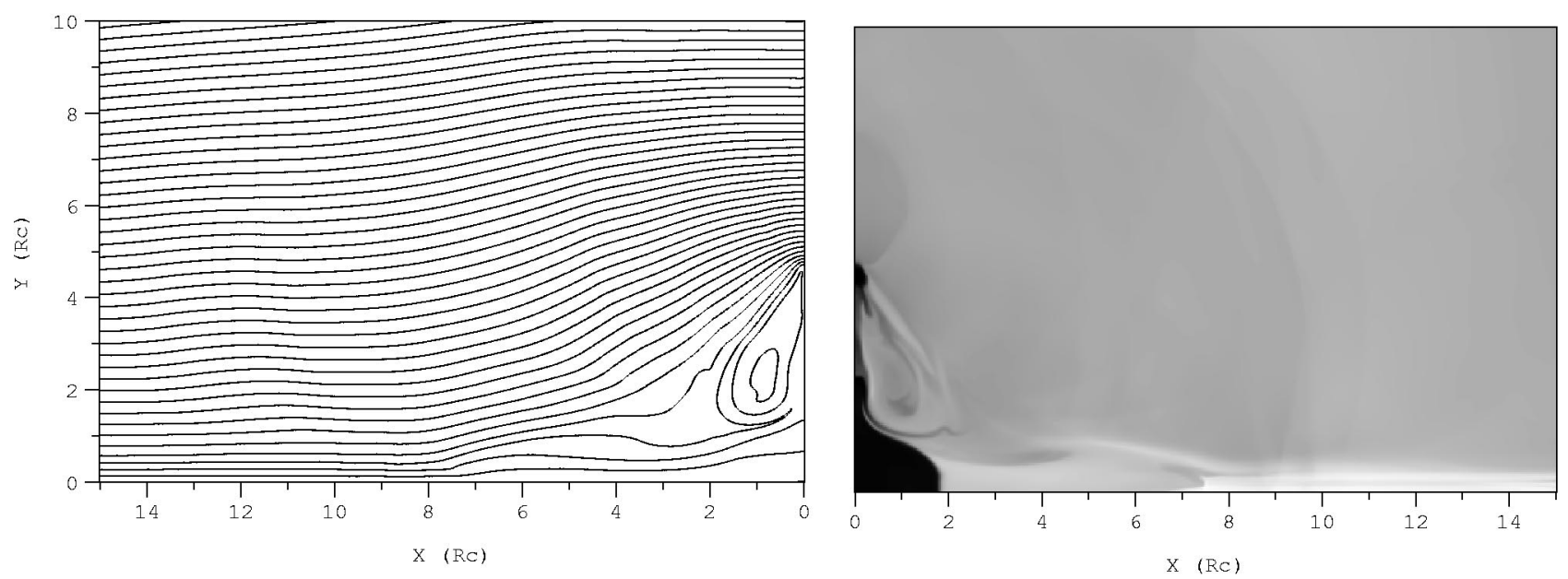

FIG. 6.-Field-line geometry (left panel) and density distribution (right panel) for case 2 at $t=30 \tau_{\text {coll }}$. Line contours and density images are as in Fig. 2 . 

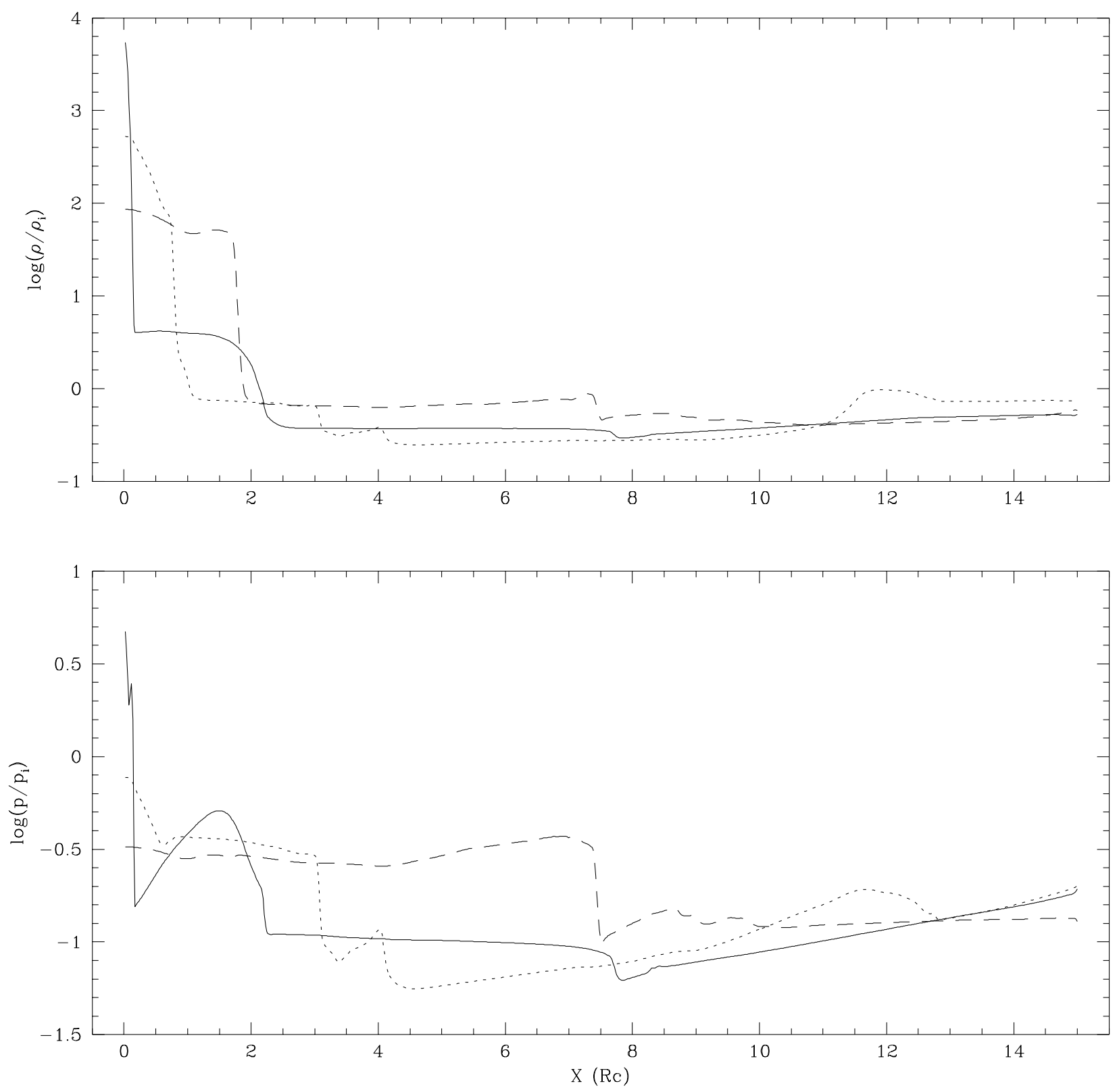

Fig. 7. - Cuts along the $X$-coordinate $\left(y=0.1 R_{c}\right)$ for the log of the density (top panel) and the log of the thermal pressure (bottom panel) for case 2. Solid line shows $t=4.5 \tau_{\text {coll }}$, dotted line $t=17.2 \tau_{\text {coll }}$, and dashed line $t=30 \tau_{\text {coll }}$.

as magnetic pressure. Then the cloud stops, and, as the field lines reexpand, is accelerated backward and its motion reversed (Fig. 12b). Therefore, we can state that the magnetic shield acts almost like an elastic magnetic bumper. At the apex of this reversal phase, both thermal and magnetic energy peak, whereas the kinetic energy obviously is about null (Fig. 11). The latter, however, returns to about $60 \%$ of its initial value at the end of the simulation (Fig. 11), and the cloud velocity is $v_{x} \sim c_{\mathrm{si}}$. Thermal and magnetic energy are also back to their initial values. Therefore, the only effect of the collision is to dissipate part of the cloud kinetic energy, with no other major consequences.

The same qualitative result is also obtained in the radiative case 7, shown in Figures $13 a$ and $13 b$ and in Figure 11 (dotted line). Whereas the kinetic and magnetic energy evolve as in case 6 , the thermal energy and $Y_{\mathrm{cm}}$ parameter now show similar qualitative behavior but much lower values (the scale for the dotted lines in the two bottom panels of Fig. 11 is 10 times smaller than for the solid lines); thus, the evolved cloud is mostly supported by magnetic pressure. Figures $13 a$ and $13 b$ show the magnetic bumper effect for the radiative case as well. The final kinetic energy is about the same as in the previous case $(50 \%$ of the initial value). However, the cloud gas has extensively spread out, especially in the $X$-direction, creating a dense, elongated structure. Because of the high density reached by the cloud during the reversal phase, the resulting cloud shape is probably limited by the diffusivity of the code. Nevertheless, the final outcome differs from the analogous HD and previous aligned field cases, being more similar to case 6 . The cloud 

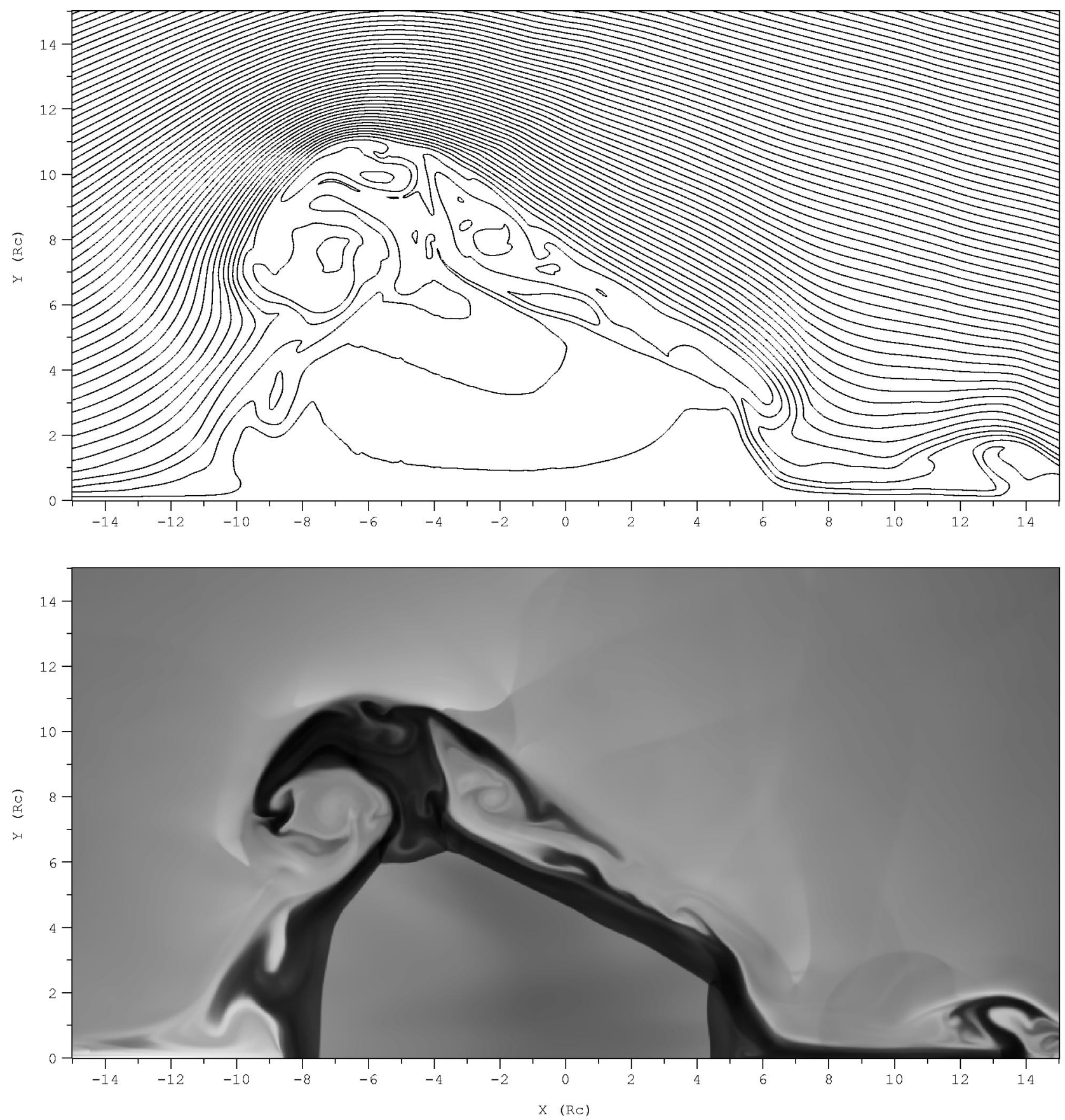

FIG. 8. - Field-line geometry (top panel) and density distribution (bottom panel) for case 3 at $t=22.5 \tau_{\text {coll }}$. Line contours and density images are as in Fig. 2 .

neither disperses nor coalesces; its structure is, however, strongly distorted and its kinetic energy partly conserved.

\section{SUMMARY AND DISCUSSION}

We have investigated the role of the magnetic field in CCs through high-resolution, fully MHD, two-dimensional numerical simulations. This paper is an extension of the gasdynamical study presented in Paper I. Our aim is to provide a first step toward the understanding of the physical role of magnetic fields in interstellar diffuse CCs. In particular, we have studied magnetic influences on (1) the final fate of the cloud after the collision (i.e., dispersal, coagulation, shattering, and filamentation); (2) the evolution of cloud kinetic energy, and (3) the effects of CCs on the mag- netic field structure in and around the clouds. These simulations represent only an initial attempt to study a complex problem. To identify the most obvious and simplest behaviors, we have restricted the geometrical freedom of the flow to two dimensions. For all behaviors, but particularly for (3), our results need to be confirmed by more thorough and extended three-dimensional calculations. The main results can be summarized as follows:

1. Adiabatic, aligned-field CCs are disruptive (as in the HD case) for both symmetric and asymmetric events. The remnant consists of an elongated structure of low magnetic energy, in which cloud and background gas are mixed together.

2. Addition of an aligned field to radiative, symmetric 

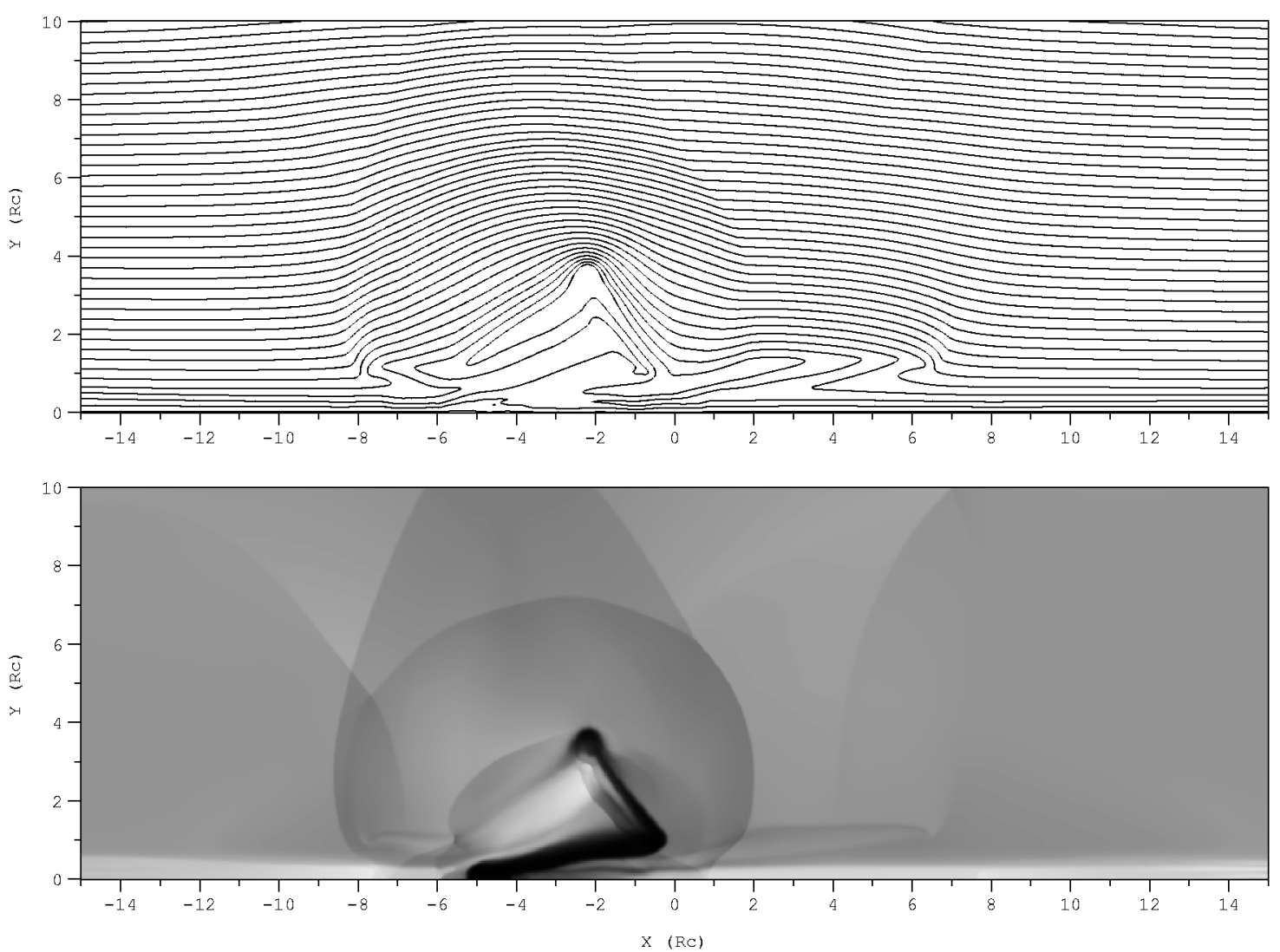

FIG. $9 a$
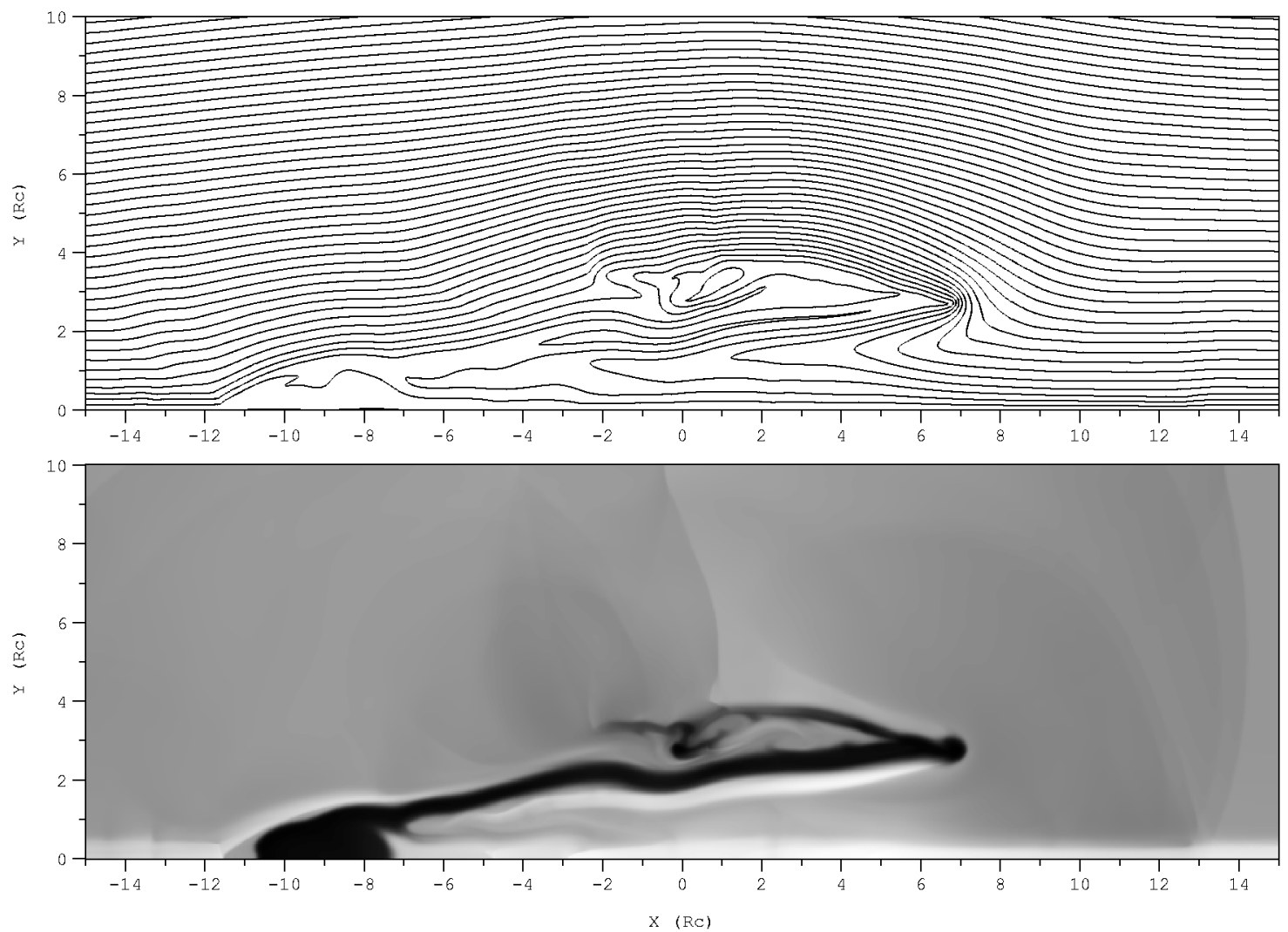

FIG. $9 b$

FIG. 9. - Field-line geometry and density distribution for case 4 at $(a) t=6.75 \tau_{\text {coll }}$ and $(b) t=22.5 \tau_{\text {coll }}$. Line contours and density images are as in Fig. 2 . 

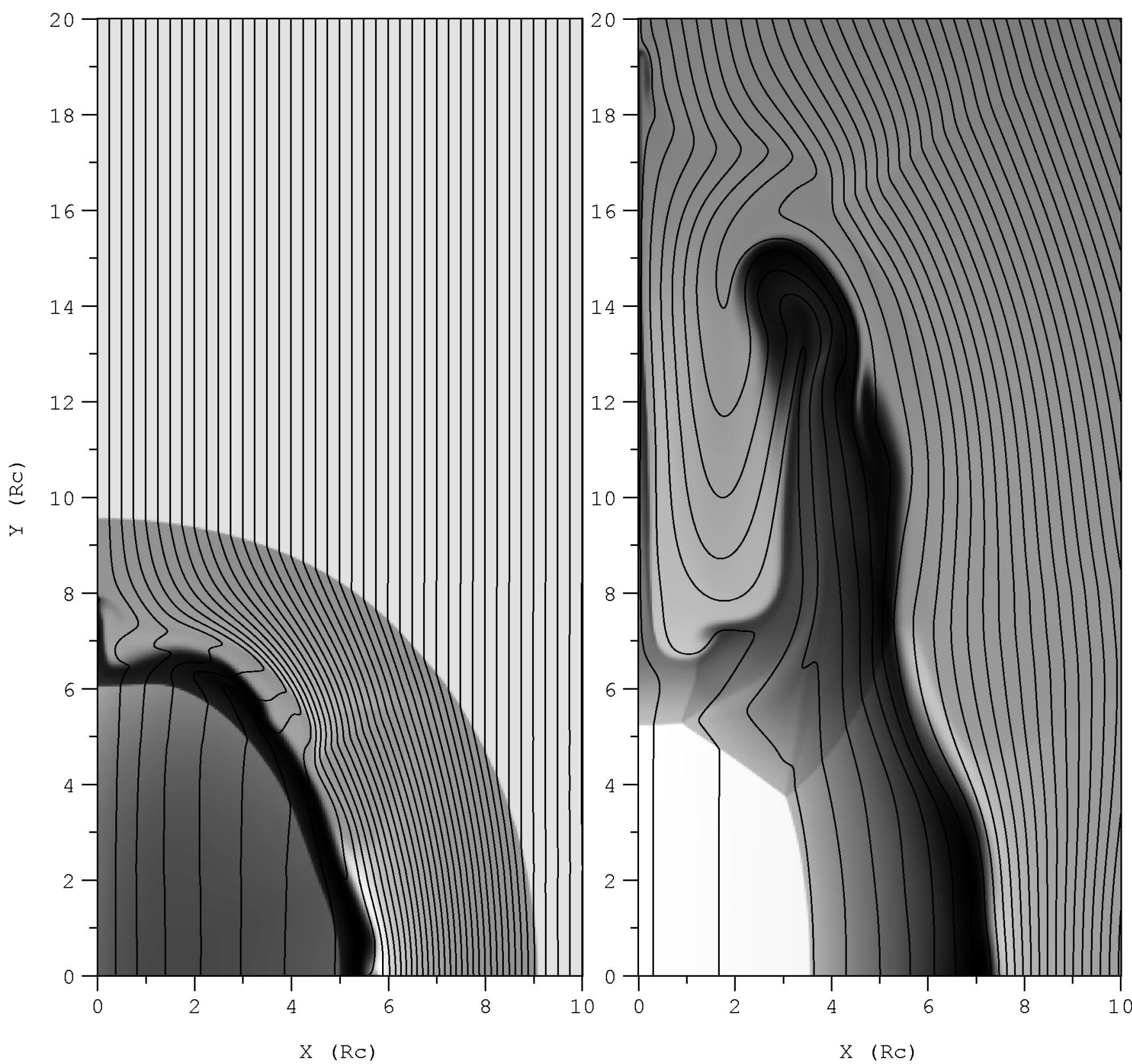

Fig. 10.-Density distribution with superimposed field-line geometry for case 5 at $t=7.5 \tau_{\text {coll }}$ (left panel) and $t=30 \tau_{\text {coll }}$ (right panel). Line contours and density images are as in Fig. 2.

CCs does not change the fact that they dissipate most of the cloud kinetic energy, which leads to almost complete coalescence of the two clouds. During asymmetric collisions of this type coagulation takes place as well, but the final structure has a mass only slightly larger $(\sim 10 \%$ for case 4$)$ than either cloud initial mass; little alteration of the magnetic-field line pattern is seen. This result is important, since purely HD asymmetric collisions of diffuse clouds have been shown to be highly disruptive (Paper I; Klein et al. 1995 and references therein).

3 . In two dimensions, motion of clouds moving transverse to the magnetic field leads to the formation of a magnetic shield in front of each cloud. When two evolved clouds of that kind run into each other, a magnetic shield may prevent direct collision from taking place. In our simula- tions, the clouds remain separated by a magnetic barrier and bounce back with a fraction $\epsilon$ of the initial kinetic energy. According to our results, $\epsilon \sim 0.5-0.6$ for both the adiabatic and radiative cases. This is probably an upper limit in more realistic situations, including threedimensional, and especially off-axis collisions, since the magnetic bumper may be less developed and other degrees of freedom (e.g., rotation) are available. In addition, if a third dimension were included, after the collision of the clouds the compressed magnetic shield would partially reexpand perpendicular to the direction of the initial motion, thus reducing the $\epsilon$ value further.

Despite the caveats mentioned above, much of the character represented in the last point may be independent of the symmetry of the collision. This was tested in part 

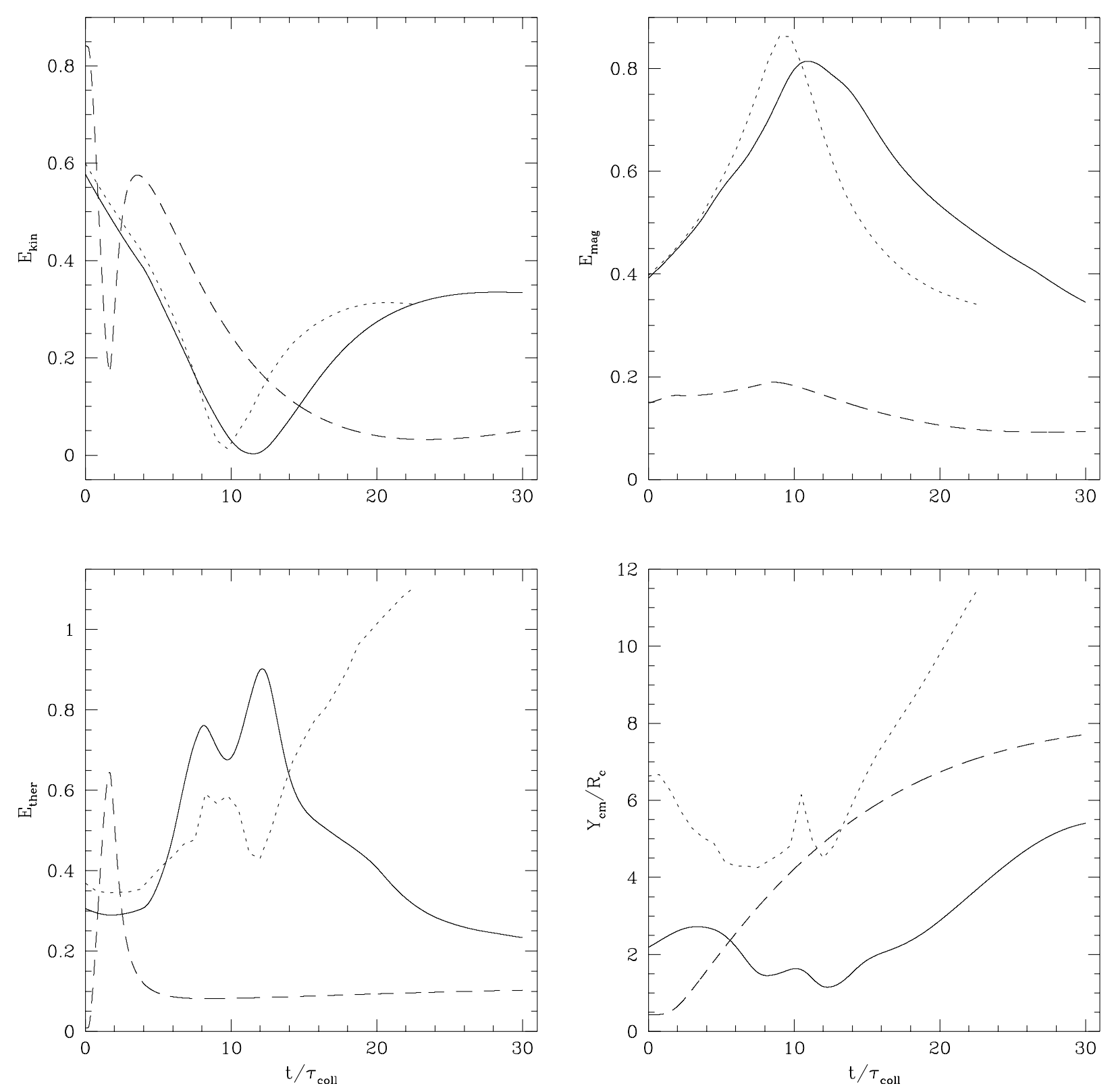

Fig. 11. - BY plots; as in Fig. 1, but for transverse field cases. Dashed lines show case 5, solid and dotted lines cases 6 and 7, respectively. The latter two lines have been multiplied by a factor 10 and 100, respectively, in order to make them readable on the same vertical scale as the dashed line.

through a low-resolution two-dimensional numerical experiment of an asymmetric, head-on, transverse-field collision. Results were consistent with those cited. In general, the magnetic shield is expected to work at some level for off-axis and largely asymmetric cases; transfer of momentum and angle scattering would occur in a manner similar to head-on collisions. When the magnetic field is aligned with the cloud motion, some of the arguments discussed in Paper I for off-axis HD CC should apply here as well. In particular, off-axis CCs with an impact parameter $b \ll R_{c}$ should be well represented by our asymmetric cases. For adiabatic cases, we also expect that even for $b \gtrsim R_{c}$, CCs should produce a reexpansion that is strong enough to disperse the clouds. The asymmetric radiative case results, however, caution us from extending the HD results to radiative MHD CC when $b$ is comparable to $R_{c}$. Those cases must be investigated in the future. Finally, for $b \sim 2 R_{c}$ the collision should produce only minor perturbation to the clouds.

A striking difference exists between the outcome of CCs when aligned and transverse field geometries are considered. Therefore, in order to correctly model CCs in the ISM it is important to understand the conditions for the formation of the magnetic bumper. Two points are crucially important in this regard: the assumed cloud shape and the initial configuration.

As for the former instance, no three-dimensional MHD individual supersonic cloud numerical simulation has been published so far. Particularly near the nose of the clouds, we should expect to see some kind of magnetic shield develop. On the other hand, divergence of the flow away from the nose and transverse to the prevailing field orientation 

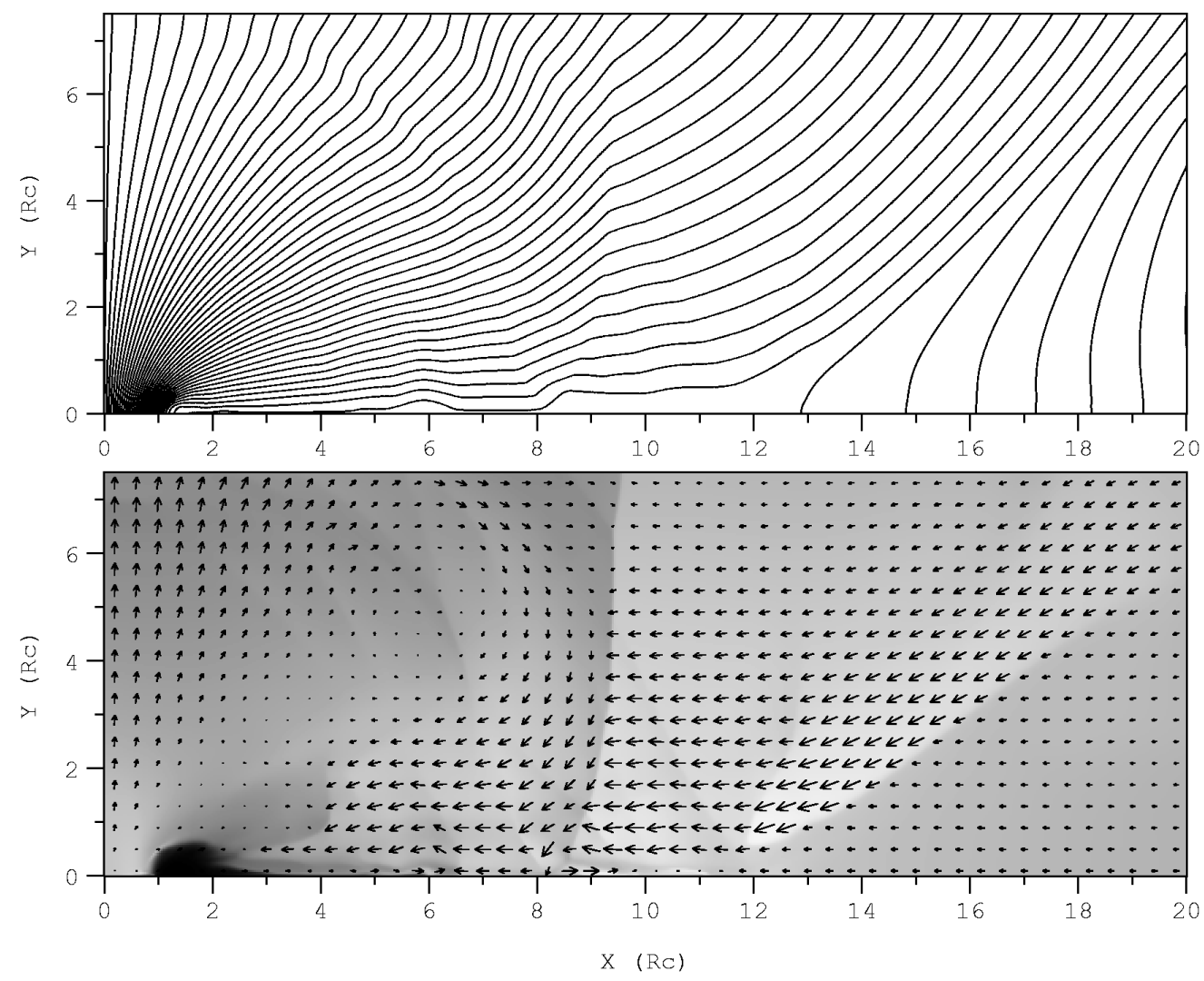

FIG. $12 a$
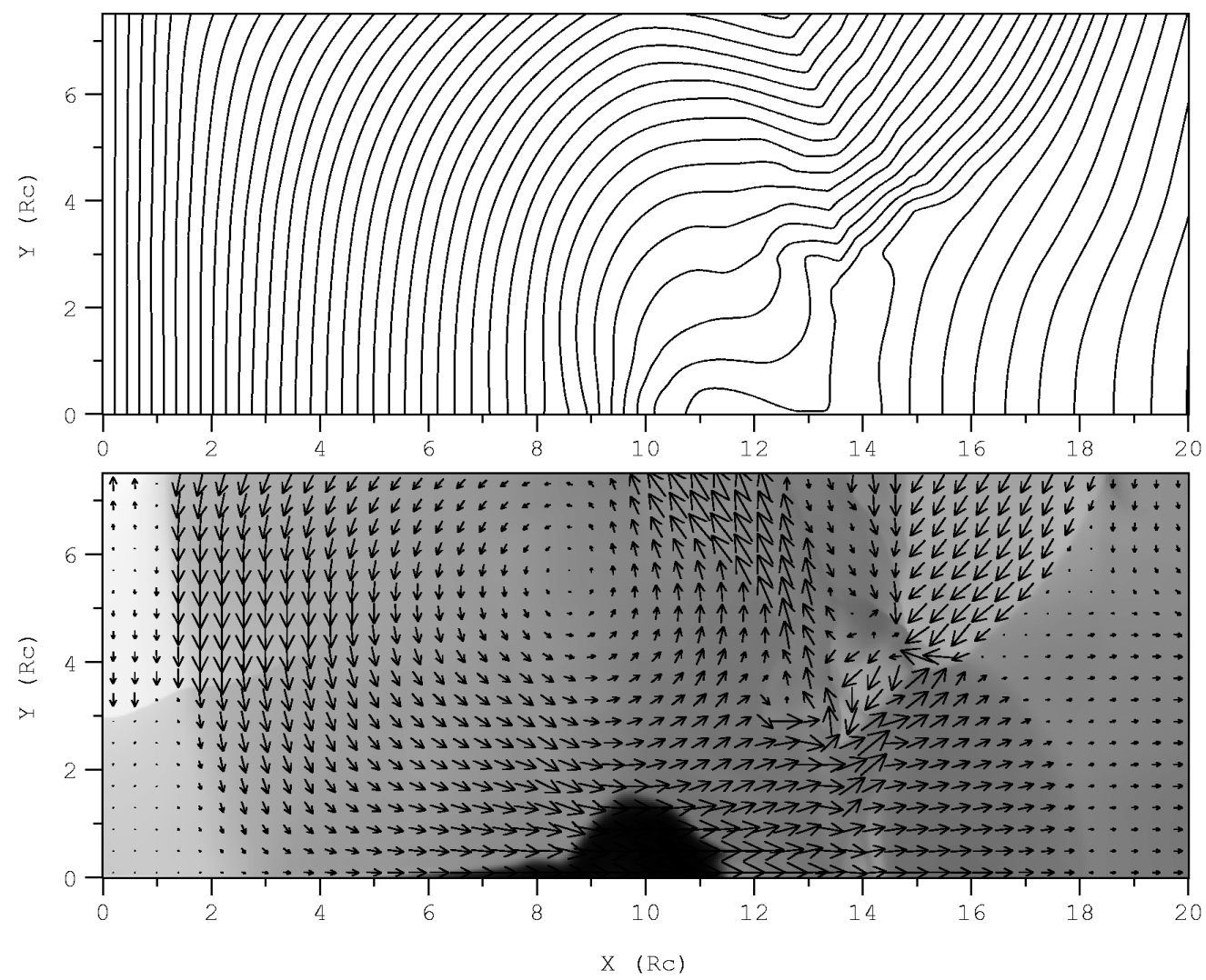

FIG. $12 b$

FIG. 12.-Field-line geometry and density distribution for case 6 at $(a) t=12 \tau_{\text {coll }}$ and $(b) t=30 \tau_{\text {coll }}$, respectively. Note that since the magnetic field is significantly stronger than in previous cases, contours of the magnetic flux (for the field lines) correspond to a change in the latter by a factor of 14 (instead of 7 as before). Density images, on the other hand, are as in Fig. 2. 

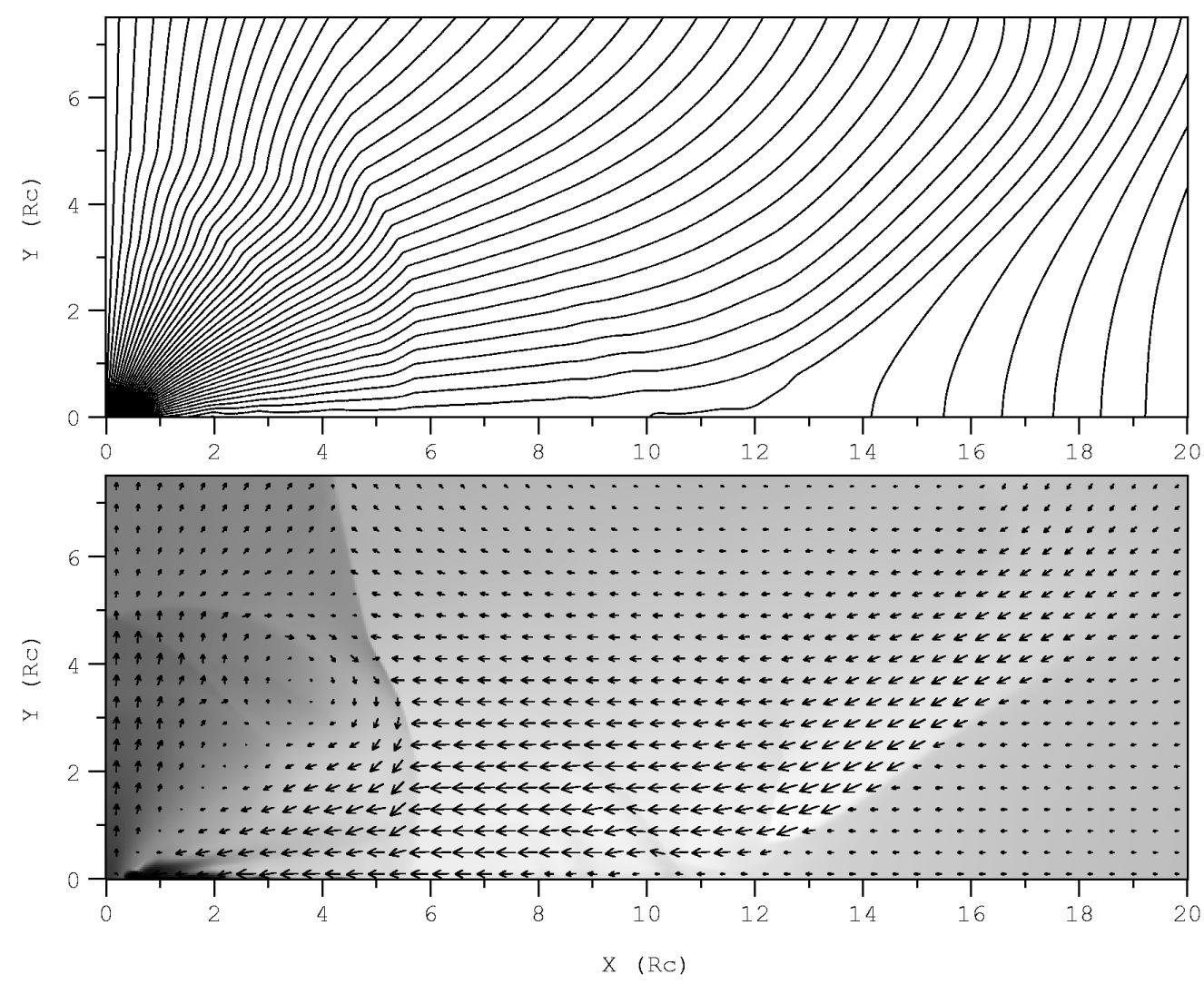

FIG. 13a
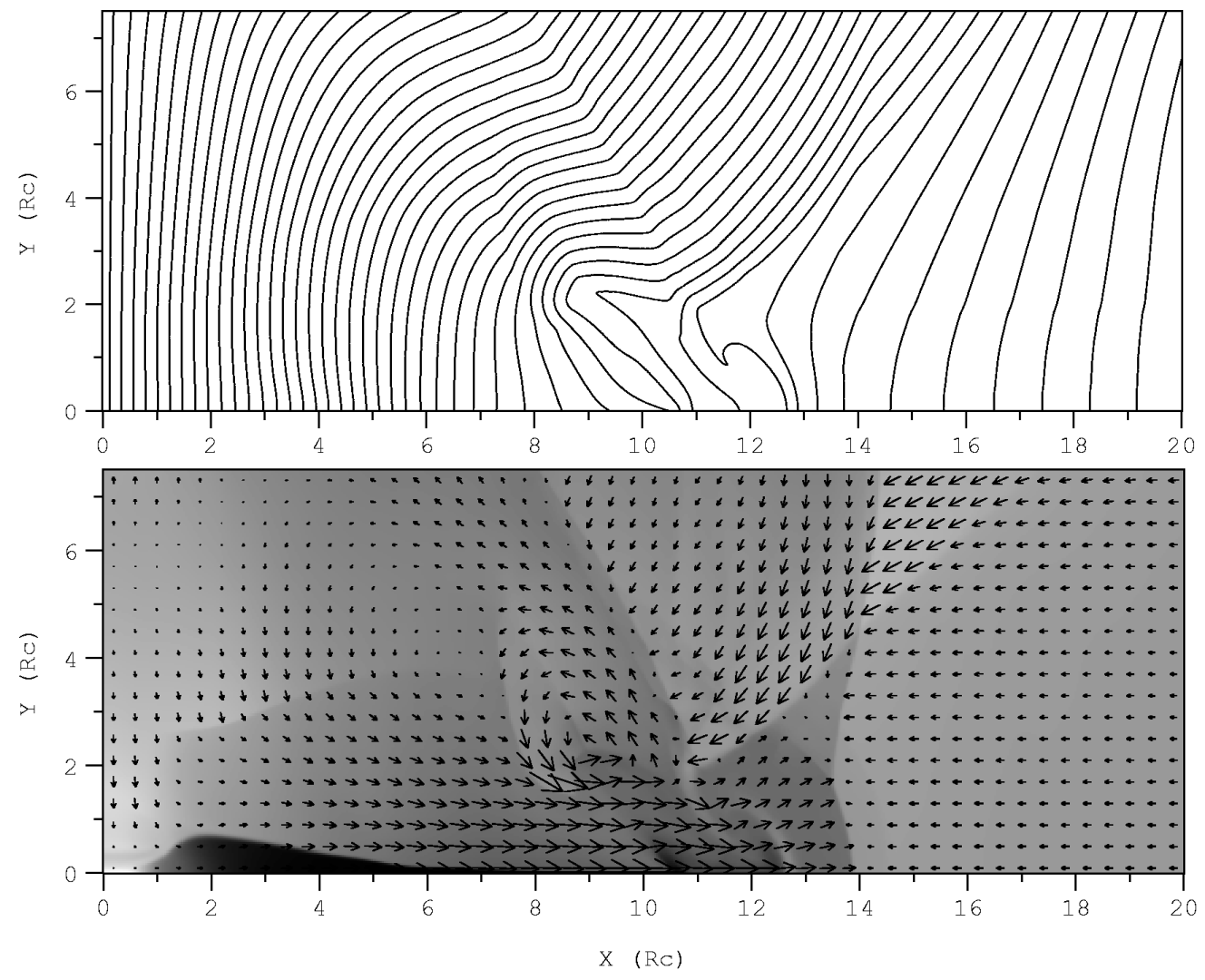

FIG. $13 b$

FIG. 13.-Field-line geometry and density distribution for case 7 at $(a) t=9.75 \tau_{\text {coll }}$ and $(b) t=22.5 \tau_{\text {coll }}$, respectively. Line contours and density images are as in Fig. 12. 
should advect field lines away from the nose, thus limiting its development and extent. The importance of that effect will depend on the geometry of the cloud. A "pointed cloud" will have only a very limited shield. For example, Koide et al. (1996), studying the propagation of extragalactic jets through a medium with an oblique magnetic field, find that the field lines distort to let the jet pass through. This effect reduces the strength of the magnetic shield and could be important for spherical clouds. However, for cylindrical or filamentary clouds, with axes transverse to the motion, field lines may be trapped at the front of the cloud long enough to play a major dynamical role. We point out that elongated clouds are not simply convenient to our two-dimensional approximation. Rather, elongated shapes are expected for clouds that form in a magnetized environment, where the support provided by the magnetic pressure is anisotropic (e.g., Spitzer 1978). As already pointed out by Jones et al. (1996), realistic clouds show strong shape irregularities where the field lines can penetrate, be captured, and stretched to form some sort of magnetic bumper. But even if this is not the case, when a cylindrical cloud moves in a transverse magnetic field, the motion of the gas along the cloud major axis, away from the stagnation point, is certainly slower at the center of the cloud, where the stagnation point is located, than near the sides. Since the field lines are frozen into the gas, the central region of the cylindrical cloud is where the field lines are held longest. Therefore, an uneven magnetic tension is applied on the cloud and a bending is produced in the central region. As a result, it becomes more difficult for the field lines to slip by the cloud, enhancing the deformation of the cloud and increasing the trapping of the field lines.

Finally, we have just begun a set of preliminary lowresolution numerical calculations, to be presented in a subsequent paper (Gregori et al. 1998). From those calculations we can anticipate that a magnetic shield always forms, and in a fashion qualitatively similar to that seen in twodimensional cases. Moreover, as expected, initially elongated clouds develop a stronger magnetic shield than spherical clouds. However, in the latter case the tension of the magnetic field lines wrapped around the cloud produces a strong deformation of the cloud shape, which grows strongly elongated transverse to the plane containing the field and the motion, thus facilitating the formation of the magnetic shield.

On the other hand, the effect of the initial configuration on individual two-dimensional cloud evolution has been investigated by Miniati et al. (1998), who study the influence of the initial field orientation with respect to the cloud motion $(\theta)$, and of the cloud density contrast $(\chi)$ and velocity $(M)$ on the magnetic bumper formation. They find that as long as two-dimensional approximation is valid and $\theta \gtrsim$ $30^{\circ}$, the timescale for the formation of the magnetic bumper is of the order of $\tau \sim(\beta \chi)^{2 / 3} M^{4 / 3} \tau_{\text {coll }}$. Since clouds are slowed down by the ram pressure of the impinging flux on a timescale of $\tau_{\mathrm{de}} \sim \chi \tau_{\text {coll }}$, they also concluded that magnetic bumpers are more likely to develop around high density contrast, low Mach number clouds.

Another important three-dimensional issue is the reexpansion of cloud gas in the direction perpendicular to the computational plane (along the $Z$-axis). This effect is important because it could in principle modify our previous conclusions for the nondisruptive cases. However, as it turns out, this only sets a limit on the length of the cloud major axis for the adiabatic case 6 . In radiative cases, lateral reexpansion involves only a small fraction of the cloud mass, independent of the $Y$ or $Z$ direction (see $\S 4.1 .1$ ). On the other hand, in the adiabatic case, the rarefaction wave generating such reexpansion propagates at the sound speed of the postshock gas, whose temperature has been enhanced by a factor of $\sim M^{2}$. Therefore, $v_{\exp } \sim M c_{\mathrm{si}}$. If the length of the cylinder is $\ell_{z}=\mu R_{c}$, the reexpansion occurs on a timescale $\tau_{\exp } \simeq \ell_{z} / v_{\exp } \sim \mu R_{c} /\left(M c_{\mathrm{si}}\right)=\mu \tau_{\text {coll }}$. Consequently, since $\tau_{\text {rad }} / \tau_{\text {exp }}=\tau_{\text {rad }} / \mu \tau_{\text {coll }}=\eta / \mu$, then if $\mu \gg 1\left(\ell_{z} \gg R_{c}\right.$ for cylindrical clouds), the cloud behaves as it did in the twodimensional radiative cases discussed before.

The aforementioned reasons justify two-dimensional simulations as a valuable starting point for the more complex three-dimensional MHD CCs. As already mentioned, however, in three-dimensional models we expect to observe new behaviors not seen in two-dimensional calculations. We expect those to be mostly related to differences in the evolution of the magnetic field. Since the magnetic field is dynamically dominant in transverse field cases, new behaviors will be more apparent there. For example, even when the magnetic shield forms in three dimensions as well, if its strength is much less than in two dimensions, then we can expect results different from those reported in this paper (see § 4.2). Nevertheless, instabilities, and in general flows along the third direction, as well as quantitative differences in cloud features developed in two and three dimensions, will certainly affect the dynamics of the collision. We have begun a series of three-dimensional MHD cloud simulations to address these complex issues more fully.

F. M. devotes his efforts in this work to the memory of his friend, Leonardo Muzzi, young artist of deep perspective, who inspired his way of studying science. We thank R. Dgani for insightful discussions on the dynamics of magnetized flows, J. Vallée for useful comments about the observed properties of the magnetic field, and E. Vazquez Semadeni for stimulating discussions. The work by F. M. and T. W. J. is partially supported by NSF grants AST 93-18959 and INT-9511654 and by the University of Minnesota Supercomputer Institute. The work by D. R. was supported in part by KOSEF through grants 975-0200006-2 and 981-0203-011-2. A. F. acknowledges the hospitality of the University of Minnesota, where this work was started.

\section{APPENDIX A}

We discuss here some details of the treatment of the radiative losses and of the mass-tracing routine. Radiative losses have been taken into account using the same approach as in Paper I, to which we refer for a detailed description. The radiative correction that we have applied is quasi-second-order accurate (see, e.g., LeVeque 1997 for a general discussion). Let us 
symbolically represent the numerical equation as

$$
\mathscr{D}_{t} \boldsymbol{q}=\mathscr{D}_{s}(\boldsymbol{q}),
$$

where $\mathscr{D}_{t}$ and $\mathscr{D}_{s}$ are the temporal operator and the spatial plus source operator, respectively, and $q$ is the set of variables describing our system. A second-order-accurate splitting from time step $n$ to $n+2$ for the operator $\mathscr{D}_{s}$ would be (Strang 1968)

$$
S_{1 / 2} L_{x} L_{y} S_{1 / 2} S_{1 / 2} L_{y} L_{x} S_{1 / 2}
$$

where $L_{x}$ and $L_{y}$ are the differential operator with respect to the $X$-coordinate and $Y$-coordinate, respectively, and $S$ is the operator representing general source terms. The subscript $1 / 2$ means that the operator is applied for only half of a time step. Instead, we have used

$$
S_{1 / 2} L_{x} L_{y} S L_{y} L_{x} S_{1 / 2},
$$

which assumes that $S_{1 / 2} S_{1 / 2} \equiv S$. Hence, the "quasi-second-order" description. In addition, we have suppressed cooling inside the shock thickness. Indeed, in the physical shock layer the flow should be nonradiative, because the crossing time of the real shock thickness (artificially spread out by the code) is much shorter than the cooling time. In addition, since density and pressure are not accurate inside the shock, but only adjacent to it, radiative cooling could become artificially large, reducing the performance of the code. This turns out to be of particular importance for the MHD calculation. The radiative cooling function we have used is identical to that in Paper I and is fully described in Ferrara \& Field (1994). It includes cooling due to free-free emission, recombination lines, and collisional excitation lines, as well as heating terms provided by collisional ionization and ionization by cosmic rays. For the cosmic-ray ionization rate, we adopt the value $2 \times 10^{-17} \mathrm{~s}^{-1}$, as determined from observations by van Dishoeck \& Black (1986). This and other rates can be found in Ferrara \& Field (1994) and references therein. We neglect dust, particularly PAHs, and photoelectric heating. This is certainly a rough approximation as far as an accurate model of the multiphase ISM is concerned (e.g., Wolfire et al. 1995). However, our aim here is to build a simple, albeit reasonable, model for the two-phase ISM and concentrate on the properties of collisions that do not depend drastically on the details of the multiphase structure. When pressure equilibrium is imposed, a two-phase (cloud + intercloud) ISM structure results.

Finally, a routine based on van Leer's second-order advection scheme (van Leer 1976) has been included to track the fraction of cloud material inside each grid cell. This quantity, referred to as the "mass tracer" or "mass fraction" (Xu \& Stone 1995), is initially set to unity inside the cloud and zero elsewhere. The mass fraction allows us to discriminate between the different components in our simulations, which are the two clouds and the intercloud medium. In this way we can calculate various quantities of interest, such as each cloud's kinetic and thermal energy, as well as $Y_{\mathrm{cm}}$, the $Y$-coordinate of the center of mass of each cloud (Jones et al. 1996). These are used in the analysis of our results.

\section{REFERENCES}

Biskamp, D. 1993, Nonlinear Magnetohydrodynamics (Cambridge: Cambridge Univ. Press)

Chandrasekhar, S. 1961, Hydrodynamic and Hydromagnetic Stability (Oxford: Clarendon)

Clifford, P., \& Elmegreen, B. G. 1983, MNRAS, 202, 629

Dai, W., \& Woodward, P. R. 1998, ApJ, 494, 317

Evans, C. R., \& Hawley, J. F. 1988, ApJ, 332, 659

Ferrara, A., \& Field, G. B. 1994, ApJ, 423, 665

Field, G. B., \& Saslaw, W. C. 1965, ApJ, 142, 568

Frank, A., Jones, T. W., Ryu, D., \& Gaalaas, J. B. 1996, ApJ, 460, 777

Gilden, D. L. 1984, ApJ, 279, 335

Gomez De Castro, A. I., Pudritz, R. E., \& Bastien, P. 1997, ApJ, 476, 717

Gregori, G., Miniati, F., Ryu, D., \& Jones, T. W. 1998, in preparation

Habe, A., Ikeuchi, S., \& Tanaka, Y. D. 1981, PASJ, 33, 23

Hausman, M. A. 1981, ApJ, 245, 72

Heiles, C. 1989, ApJ, 336, 808

Ikeuchi, S. 1988, Fund. Cosm. Phys., 12, 255

Jones, T. W., Gaalaas, J. B., Ryu, D., \& Frank, A. 1997, ApJ, 482, 230

Jones, T. W., Kang, H., \& Tregillis, I. L. 1994, ApJ, 432, 194

Jones, T. W., Ryu, D., \& Tregillis, I. L. 1996, ApJ, 473, 365

Jungwiert, B., \& Palous, J. 1996, A\&A, 311, 397, 306

Klein, R. I., McKee, C. F., \& Woods, D. T. 1995, in ASP Conf. Ser. 80, The Physics of Interstellar Medium and Intergalactic Medium, ed. A. Ferrara et al. (San Francisco: ASP), 366

Koide, S., Sakai, J.-I., Nishikawa, K.-I., \& Mutel, R. L. 1996, ApJ, 464, 724

Lattanzio, J. C., \& Henriksen, R. N. 1988, MNRAS, 232, 565

Lattanzio, J. C., Monaghan, J. J., Pongracic, H., \& Schwarz, M. P. 1985, MNRAS, 215, 125

LeVeque, R. J. 1997, in Astrophysical Fluid Flows, 27th Saas-Fee Advanced Course Lecture Notes (Berlin: Springer), in press

Little, L. T., Riley, P. W., Macdonald, G. H., \& Matheson, D. N. 1978, MNRAS, 183,805

Loren, R. B. 1976, ApJ, 209, 466

Mac Low, M., McKee, C. F., Klein, R. I., Stone, J. M., \& Norman, M. L. 1994, ApJ, 433, 757

Malagoli, A., Bodo, G., \& Rosner, S. 1996, ApJ, 456, 708
Melrose, D. B. 1986, Instabilities in Space and Laboratory Plasmas (Cambridge: Cambridge Univ. Press)

Meyerdierks, H. 1992, A\&A, 253, 515

Miniati, F., Jones, T. W., Ferrara, A., \& Ryu, D. 1997, ApJ, 491, 216 (Paper I)

Miniati, F., Jones, T. W., \& Ryu, D. 1998, ApJ, submitted

Murray, S. D., White, S. D. M., Blondin, J. M., \& Lin, D. N. C. 1993, ApJ, 407, 588

Myers, P. C., Goodman, A. A., Güsten, R., \& Heiles, C. 1995, ApJ, 442, 177

Myers, P. C., \& Khersonsky, V. K. 1995, ApJ, 442, 186

Norman, C. A., \& Ferrara, A. 1996, ApJ, 467, 280

Oort, J. M. 1954, Bull. Astron. Netherlands, 12, 177

Ricotti, M., Ferrara, A., \& Miniati, F. 1997, ApJ, 485, 254 (RFM)

Rohlfs, R., Herbstmeier, U., Mebold, U., \& Winnberg, A. 1989, A\&A, 211, 402

Ryu, D., \& Jones, T. W. 1995, ApJ, 442, 228

Ryu, D., Jones, T. W., \& Frank, A. 1995, ApJ, 452, 785

Ryu, D., Miniati, F., Jones, T. W., \& Frank, A. 1998, ApJ, 509, in press

Schiano, A. V. R., Christiansen, W. A., \& Knerr, J. M. 1995, ApJ, 439, 237

Smith, J. 1980, ApJ, 238, 842

Spitzer, L. 1978, Physical Processes in the Interstellar Medium (New York: Wiley)

Stone, M. E. 1970a, ApJ, 159, 277

.1970b, ApJ, 159, 293

Strang, G. 1968, SIAM J. Numer. Anal., 5, 506

Struck-Marcell, C., \& Scalo, J. M. 1984, ApJ, 277, 132

Theis, C., Burkert, A., \& Hensler, G. 1992, A\&A, 265, 465

Vallee, J. P. 1995, AJ, 110, 2256

van Dishoeck, E. F., \& Black, J. H. 1986, ApJS, 62, 109

van Leer, B. 1976, J. Comput. Phys., 23, 276

Vázquez, E., \& Scalo, J. M. 1989, ApJ, 343, 644

Vietri, M., Ferrara, A., \& Miniati, F. 1997, ApJ, 483, 262

Wolfire, M. G., Hollenbach, D., McKee, C. F., \& Tielens, A. G. G. M. 1995, ApJ, 443, 152

Xu, J., \& Stone, J. M. 1995, ApJ, 454, 172

Zeldovich, Ya. B., Ruzmaikin, A. A., \& Sokoloff, D. D. 1983, Magnetic Fields in Astrophysics (New York: Gordon \& Breach) 